\title{
Security, Privacy, and Usability in Continuous Authentication: A Survey
}

\author{
Ahmed Fraz Baig ${ }^{1,2, *,+}$ and Sigurd Eskeland ${ }^{1,+}$ \\ 1 Norwegian Computing Center, 0373 Oslo, Norway; sigurd@nr.no \\ 2 Department of Information Security and Communication Technology, Norwegian University of Science and \\ Technology, 2815 Gjøvik, Norway \\ * Correspondence: baig@nr.no \\ + These authors contributed equally to this work.
}

Citation: Baig, A.F.; Eskeland, S. Security, Privacy, and Usability in Continuous Authentication: A Survey. Sensors 2021, 21, 5967. https:/ / doi.org/10.3390/s21175967

Academic Editors: Weizhi Meng, Rongxing Lu and Evangelos Kranakis

Received: 18 June 2021

Accepted: 26 August 2021

Published: 6 September 2021

Publisher's Note: MDPI stays neutral with regard to jurisdictional claims in published maps and institutional affiliations.

Copyright: (c) 2021 by the authors. Licensee MDPI, Basel, Switzerland. This article is an open access article distributed under the terms and conditions of the Creative Commons Attribution (CC BY) license (https:// creativecommons.org/licenses/by/ $4.0 /)$.

\begin{abstract}
Continuous authentication has been proposed as a possible approach for passive and seamless user authentication, using sensor data comprising biometric, behavioral, and contextoriented characteristics. Since these are personal data being transmitted and are outside the control of the user, this approach causes privacy issues. Continuous authentication has security challenges concerning poor matching rates and susceptibility of replay attacks. The security issues are mainly poor matching rates and the problems of replay attacks. In this survey, we present an overview of continuous authentication and comprehensively discusses its different modes, and issues that these modes have related to security, privacy, and usability. A comparison of privacy-preserving approaches dealing with the privacy issues is provided, and lastly recommendations for secure, privacy-preserving, and user-friendly continuous authentication.
\end{abstract}

Keywords: continuous authentication; security; privacy; usability; user experience

\section{Introduction}

We are dependent on computing technology to store and process our personal data. We interact with devices in the form of smart-phones, cars, sensors, Internet of Things (IoT), and other devices. Authentication ensures that the given entity is one it claims to be [1]. Authentication can be characterized by different factors such as knowledge-based authentication (PIN, password), possession-based (devices, smartcards, etc.), physiologicalbased (such as fingerprint, iris, voice, face), behavioral-based (such as keystroke dynamics, touch dynamics, motion dynamics, etc), and context-aware factors (such as physical location, IP-addresses, device-specific data, browsing history, etc.). According to a report [2], cyberattacks are happening every year, and accounts are being compromised every second. This happens due to poor implementation of authentication mechanisms. Weak passwords can be broken easily and strong passwords are not memorable. Nowadays, physiological biometric-based approaches are widely adopted in smart devices that use face recognition and fingerprints recognition, which are examples of what we refer to as authentication modes or simply "modes". These approaches attempt to improve the usability over conventional authentication approaches. A disadvantage about physiological biometrics is that physiological features are static, which can be reproduced by an adversary.

In general, user authentication can be performed on a device or a server-side: (1) The user will authenticate himself towards the mobile device. Device-side authentication is performed entirely on the device [3]. (2) The user will authenticate himself towards a cloud service. By means of his user credentials, the user proves his authenticity to the cloud (authentication server), which performs the user authentication and grants the user access to the service if the authentication succeeded. High-performance computational resources and on-demand availability enable users and companies to leverage cloud-based services. Many mobile devices are using cloud-based services for data processing and 
storage, which facilitate access to multiple services and also provide easy backup to data. In a "traditional" setting, the user authenticates himself at the beginning of a session. In the case of device-side authentication, the session normally lasts as long as the user is using the device actively, and after that, it locks automatically. In the case of service/cloud-side authentication, the user provides some authentication credentials to the server, which then determines whether the user is authentic or not and on that basis grants the user access to the service.

A potential problem about session-oriented approaches is that if the user leaves the computer or the device for some time, a malicious user accessing the device in the meantime is not prevented from using it or any services that the user is logged onto. This issue could be mitigated by security mechanisms that continuously re-authenticate the user during the session. User authentication can be conducted actively or passively. The former requires explicit user attention or user action, such as entering a password or pin or putting a finger on the finger print scanner. The latter is a seamless and transparent form of authentication that runs in the background without notifying the user or requiring any user attention.

Continuous authentication (CA) offers advantages with regard to usability and security. It passively re-authenticates users without notifying the user or requiring any user attention, and it locks the system automatically in case the user is inactive or when it observes irregularities or anomalous behaviors. Continuous authentication utilizes physiological biometrics pattern recognition, behavioral biometrics pattern recognition, and context-aware authentication modes, sometimes in combination. Combining multiple modes is sometimes referred to as multi-modal authentication.

Usability pertains to ease of access, user friendliness, how satisfactorily or efficient a product or service achieves its function, and how prone it is to errors (ISO 9241-11:2018 [4]). Continuous authentication seeks to offer some trade-off between security, privacy, and usability due to its passive and seamless operation. It continuously monitors users actions and behaviors, which achieves security and usability, but not privacy as authentication mechanisms are conceptually security mechanisms. Since continuous authentication mechanisms collect personal data, such as physiological, behavioral, and context-aware user data, this causes privacy concerns.

\section{Survey Contributions}

This survey presents an overview of continuous authentication modes with performance comparison. Specifically, we seek to answer the following questions:

1. What privacy and security challenges are there when data processing and storage is conducted in the cloud versus locally on the device?

2. How do the different CA modes score with regard to privacy (disclosure of personspecific information about behavior, location, physiological biometric characteristics, etc.) and security (how secure authentication is obtained by a mode)?

3. What techniques are preferable for mitigating the privacy and security issues of the different CA modes?

4. What behavioral, physiological, and context-aware modes, and combinations thereof, are most suited in actual CA implementations?

Furthermore, besides these questions, this survey also discusses open challenges related to usability, challenges related to ISO/ICE standards, and other challenges regarding the applicability of continuous authentication in real-time projects. The rest of the survey is arranged as follows: Section 2 discusses preliminaries; Section 3 provides a detailed overview of several modes of continuous authentication; Section 4 discusses the security and privacy risks associated with different modes of continuous authentication. The usability and other open issues are discussed in Section 5. Section 6 discusses privacy-preserving approaches and provides future recommendations. Moreover, Section 7 discusses the list of related surveys conducted for continuous authentication. Finally, the conclusion of the survey is stated in the last section. 


\section{Preliminaries}

In this section, we introduce some basic authentication concepts. According to ISO/IEC 24760-1 [5], an identity is a "set of attributes related to an entity". Continuous authentication with physiological and behavioral biometrics utilizes user-specific biometric information (referred to as templates) for user identification, whereas contextaware continuous authentication modes use context-related information for authentication. The following subsections explain authentication properties and identity management.

\subsection{Properties of Identification}

The following are properties required for identification [6]:

- Uniqueness means that each subject should have a unique identity or a set of identities.

- Universality means that the unique identity is invariant for a period of time and within a predefined scope.

- Acceptability relates to user experience, to what extent that users will accept their identities, and to how applicable or practical they are.

\subsection{Steps for Authentication and Data Processing}

Continuous authentication can be accomplished with following steps:

- Data acquisition. Raw data are acquired by various devices that contain a number of sensors (such as accelerometer, proximity sensor, camera, magnetometer, gyroscope, GPS sensors, etc.). Human behavioral information can be attained by proximity sensors and accelerometers, while gyroscope sensors detect smartphone's rotational motion. GPS sensors collection location data.

- Feature extraction. Obtaining a set of useful features/attributes from the collected data.

- Feature selection. This phase removes irrelevant, redundant, and noisy features and selects only the most relevant features from the set of useful features.

- Classification. This phase divides the users into two classes that agree with the authentication outcome of acceptance or rejection. Various Machine Learning (ML) algorithms can be utilized for classification purposes, such as k-Nearest Neighbours (k-NN), Neural Network (NN), Support Vector Machine (SVM), Decision Tree (DT), and many others.

The literature on continuous authentication refers mostly to a few well-known ML algorithms, such as k-NN, SVM, NN, and DT. A brief introduction of these algorithms with their advantages and limitations is given in the following subsection.

\subsubsection{Algorithms}

In this subsection, we provide a brief introduction of the most common ML algorithms that are used for continuous authentication.

(1) $k$-Nearest Neighbors ( $k$-NN) [7]. Given $N$ feature vectors (training vectors), this algorithm identifies $k$ nearest neighbors of a point in a class. The working of $k-\mathrm{NN}$ relies on the distance between feature vectors. Nearest neighbors are found by using any distance calculation algorithms, such as the Euclidean distance algorithm and the Manhattan distance algorithm, over a positive integer $k$. This algorithm selects $N$ points and starts calculating distances with all its neighbor points. It places a point in $N$ clusters according to the nearest distance.

$k$-NN is simple and easy to implement as it does not require training steps. However, it has challenges, because it only chooses neighbors based on distance values. Moreover, $k$-NN stores entire training data in the memory, which can be a reason for slow performance on large datasets [8].

(2) Support Vector Machine (SVM) [9] is the most utilized ML algorithm. It separates data classes into two groups by drawing a hyperplane (line). This line is called the decision boundary. Any data point that lies on one side of the boundary will be classified in one class (legitimate class), and anything that lies on the other side will be classified in another class (illegitimate class). 
SVM splits classes based on their distances from one data point to the other nearest data points. SVM can be classified as a linear SVM or non-linear SVM. The linear SVM segregates data with a hyperplane with a straight boundary. In contrast, the non-linear kernel does not create straight boundaries, which implies that the non-linear algorithms utilize kernels to classify non-separable data into separable data. Many continuous authentication approaches utilize SVM for classification [10-13]. SVM can perform well on small datasets; however, like $k$-NN, it cannot perform well on large and noisy datasets [14].

(3) Neural Networks (NN) [15], also known as artificial neural networks (ANN), consist of a node layer, an input layer, one or many hidden layers, and an output layer. Every node has a weight and a threshold value that is associated with connected nodes. A node is only activated when its output value is above the threshold value. Otherwise, no data are sent to the next layer of the neural network.

NN can be further classified into following types: Feed-forward Neural Network [16], Recurrent Neural Network (RNN) [17], Multilayer Perceptron (MLP) [18], Long Short Term Memory (LSTM) [19], etc. The neural network offers many advantages, such as they store information on the entire network; they can work on incomplete information and can perform multiple jobs simultaneously [20]. Moreover, each type of NN offers distinct advantages depending on applications, such as RNN performs well on image data, LSTM is suitable for time series data, and MLP has various applications in natural language processing (NLP) and speech recognition.

(4) Decision Trees (DT) [21] is a supervised ML algorithm that builds trees by continuously splitting or classifying the input data depending on certain parameters. DT consists of a root node, internal nodes (non-leaf nodes), and leaf nodes (or terminal nodes). The root node contains complete training data, the splitting process divides decision nodes into sub-nodes over a given condition, and leaf nodes or terminal nodes are the outcomes or decisions. This algorithm recursively generates new trees from the data until it reaches a stage where it cannot further classify nodes. The leaf nodes of a decision tree contain the decisions (or classifications).

\subsubsection{Performance}

Performance indicates how accurately and securely a method achieves authentication. This is measured by means of ratios of correct acceptances (true positives, TP), correct rejections (true negatives, TN), false acceptances (false positives, FP), and false rejections (false negatives, FP) [22]:

- Accuracy is the ratio of the number of correctly matched authorized users out of all users:

$$
\text { Accuracy }=\frac{\mathrm{TP}+\mathrm{TN}}{\mathrm{TP}+\mathrm{FP}+\mathrm{TN}+\mathrm{FN}}
$$

- False Acceptance Rate (FAR) is the likelihood of incorrectly accepting an unauthorized user. This is typically stated as the ratio of the number of incorrect acceptances divided by the number of incorrect acceptances (FP) and correct rejections (TN):

$$
\mathrm{FAR}=\frac{\mathrm{FP}}{\mathrm{FP}+\mathrm{TN}}
$$

- False Rejection Rate (FRR) is the likelihood of incorrectly rejecting an authorized user. This is typically stated as the ratio of the number of incorrect rejections divided by the number of incorrect rejections (FN) and correct acceptances (TP).

$$
\mathrm{FRR}=\frac{\mathrm{FN}}{\mathrm{FN}+\mathrm{TP}}
$$

- Equal Error Rate (ERR) is the rate at which both FAR and FRR are equal. The lower the ERR value of a biometric system, the higher the accuracy of the system. 
A brief introduction to ML approaches in different modes of continuous authentication is discussed in the following sections.

\section{Modes of Continuous Authentication}

In order to define CA clearly, we mention two definitions provided in the literature. Traore [23] defines CA as "a new generation of security mechanisms that continuously monitor user behavior and use this as basis to re-authenticate them periodically ... " Lorena et al. [24] defines CA as "a security mechanism that monitors user actions at every point in time ... during a session and determines if that user is the legitimate one." These definitions are a bit limited and do not cover all aspects of CA. The first definition considers only behavioral biometrics, while the second definition does not clarify whether continuous authentication is achieved actively or passively. We propose defining continuous authentication as continuously and passively monitoring users by means of recognizing user features and actions (i.e., physiological biometrics, behavioral biometrics, or context-aware authentication modes) during a session.

\subsection{Physiological Biometrics}

Physiological biometrics (fingerprint recognition, face recognition, and iris recognition) are among the well-known and most commonly used traditional authentication modes. These modes are also utilized for continuous authentication.

\subsubsection{Face and Voice as Biometrics}

Face recognition and iris recognition can be utilized for continuous authentication. A face recognition-based biometric authentication method was presented in [10]. The authors utilized the support vector machine (SVM) for experiments and recruited 32 applicants to test the prototype. Their method achieved 3.92-7.92\% EER. In 2015, Crouse et al. [11] also proposed a face recognition-based continuous authentication method for mobile devices. This method collected face images of 10 applicants and trained SVM classification algorithm for experiments. They achieved $0.1-1 \%$ FAR, $73 \%$ TAR, and $64 \%$ accuracy.

Voice recognition can be used for continuous authentication. Feng et al. [25] propose a voice recognition method for continuous authentication. It was evaluated by means of 18 users. It achieved $97 \%$ recognition accuracy with $0.1 \%$ FPR. A list of studies on facebased and voice-based continuous authentication methods with performance comparison is shown in Table 1.

Table 1. Face and voice.

\begin{tabular}{lllll}
\hline Studies & Modality & Classification Algorithms & \# Users & Performance \\
\hline$[10]$ & Face & SVM & 32 & $3.92-7.92 \%$ EER \\
{$[11]$} & Face & SVM & 10 & $0.1-1 \%$ FAR, 73\% TAR, 64\% accuracy \\
& FBP & 12 & 80 accuracy on small-size image, $96 \%$ on \\
{$[26]$} & Face & SVM & dataset & $13-30 \%$ EER \\
{$[12]$} & Face & SVM & dataset & $94 \%$ accuracy, 0.92\% TNR \\
{$[13]$} & Face & CNN & YouTube & $0.86 \%$ EER \\
{$[27]$} & Face & SVM & 18 & $97 \%$ accuracy, $0.1 \%$ FPR \\
\hline$[25]$ & Voice & SVM & 27 & $93 \%$ accuracy, $3 \%$ FRR \\
{$[28]$} & Voice & HMM & 21 & $99 \%$ accuracy, $\%$ EER, $1 \%$ FRR \\
{$[29]$} & Voice & DTW & 15 & $88 \%$ accuracy, $15 \%$ FRR, $0.01 \%$ FRR \\
{$[30]$} & Voice & HMM & 12 & $93.3 \%$ accuracy, $1.01 \%$ EER \\
\hline$[31]$ & Voice & &
\end{tabular}

\subsubsection{ECG and EEG Features as Biometrics}

Electroencephalography (EEG) measures the electrical activities of the brain signals, and electrocardiography (ECG) measures the timing and strength of heart signals. EEG and ECG are considered as unique features that can be used for user authentica- 
tion [32]. Table 2 presents experimental results and a performance comparison of a few studies [33-37], which utilized EEG and ECG as modes for continuous authentication. Some studies combine two or more different modes (multimodal biometric) for continuous authentication such as face and fingerprint, face, iris, and voice, EEG, gait and fingerprint, EEG, eye blink, etc. Table 3 indicates performance of some multimodal biometric systems found in the literature.

Table 2. EEG-, ECG-, and eye-movement-based authentication.

\begin{tabular}{lllll}
\hline Studies & Techniques & Classification Algorithms & \# Users & Performance \\
\hline$[33]$ & EEG & FFT & 23 & $11 \%$ EER \\
{$[34]$} & EEG & FFT & 23 & $79 \%$ accuracy \\
{$[35]$} & EEG & kNN & 50 & $97 \%$ CRR \\
\hline$[38]$ & ECG & ZMCP & - & $10.10 \%$ EER, $1.57 \%$ FAR, 0.39\% FRR \\
{$[39]$} & ECG & kNN-DDM & 19 & $100 \%$ accuracy, 0.36\% EER \\
{$[40]$} & ECG & SVM & - & $84.8 \%$ accuracy, $0.2 \%$ EER \\
\hline$[41]$ & Eye movement & SRC & 20 & $88.73 \%$ accuracy, $10.61 \%$ EER \\
{$[42]$} & Eye movement & SVM & 30 & $93.1 \%$ accuracy, $6.9 \%$ EER \\
{$[43]$} & Eye movement & SVM & 22 & $3.93 \%$ EER \\
{$[44]$} & Eye movement & CNN & - & $97.95 \%$ accuracy \\
{$[45]$} & Eye blink & SVM, AB & CEW & $98.4 \%$ accuracy \\
\hline$[46]$ & BioAura & - & $1.9 \%$ EER, $7.6 \%$ FAR, 9.6-8.4\% FRR \\
\hline
\end{tabular}

Table 3. MultiModal biometrics.

\begin{tabular}{|c|c|c|c|c|}
\hline Studies & Modality & $\begin{array}{l}\text { Classification } \\
\text { Algorithms }\end{array}$ & \# Users & Performance \\
\hline [36] & EEG, gait & SVM, RNN & 6 & 63.16\% FRR with EEG, 1.9\% FRR with multiple modes \\
\hline [37] & EEG, ECG & Euclidean & 526 & $\begin{array}{l}\text { 22.97-29.36\% ERR with EEG, } 0.928-8.216 \% \text { ERR with } \\
\text { multiple modes }\end{array}$ \\
\hline [47] & Face, fingerprint & HMM & 11 & $\begin{array}{l}0.9995 \% \text { accuracy with fingerprint, } 0.970 \% \text { accuracy } \\
\text { with face }\end{array}$ \\
\hline [48] & EEG, fingerprint & NBM & 40 & 4.16\% ERR with EEG, 1.12\% ERR with fingerprint \\
\hline [49] & Face and voice & LBP, VAD & 152 & $\begin{array}{l}\text { HTER: } 11.9 \% \text { (male), } 13.3 \% \text { (female), EER: } 10.9 \% \text { (male), } \\
10.5 \% \text { (female) }\end{array}$ \\
\hline [50] & EEG, eye blink & LS & 31 & $\begin{array}{l}0.89-1.1 \% \text { ERR, } 6.71 \% \text { FAR with EEG, } 2.71 \% \text { FAR with } \\
\text { multi-mode, } 8.49 \% \text { FRR with EEG, 2.09\% FRR with } \\
\text { multi-mode }\end{array}$ \\
\hline [51] & EEG, face & BT & 6 & $90 \%$ accuracy \\
\hline
\end{tabular}

\subsection{Behavioral Biometrics}

User behavior recognition can be utilized for user authentication. The following modes of behavioral biometrics are used for continuous authentication.

\subsubsection{Motion Dynamics}

Motion dynamics are indicated by the patterns of a person's gait or walking style. Gait-based recognition techniques can identify and differentiate human activities based on walking style. Motion dynamics data are collected from sensors, such as gyroscopes or accelerometers that are attached to the human body for data collection. Derawi et al. [52] used a Google mobile device (G1) containing embedded sensors for data collection. In this study, 51 volunteers participated in the data collection process by carrying mobile phones that had a motion sensor on the right-hand side of the hip. Hence, this method used Dynamic Time Warping (DTW) for matching and achieved 20\% EER. Mäntyjärvi et al. [53] placed a sensor on the waist. They performed experiments with 36 participants and utilized 
FFT for matching. The proposed method achieved an accuracy of $72-88 \%$ with $7 \%$ EER. Gafurov et al. [54] attached sensors on 100 participants, whereof 30 users had sensors on the ankle, 30 users on their arms, 100 users had sensors on the hips, and 50 participants had a mobile device in their pockets. Authors utilized kNN for classification and achieved equal error rates $(5 \%, 10 \%, 13 \%$, and $7.3 \%)$, respectively. Table 4 presents a comparison of few recent gait-based recognition approaches with respect to their performance [55-61].

Table 4. Gait-based authentication.

\begin{tabular}{llll}
\hline Studies & Classification Algorithm & \# Users & Performance \\
\hline$[52]$ & DTW & 51 & $20 \%$ EER \\
{$[53]$} & FFT & 36 & $72-88 \%$ accuracy, $7 \%$ EER \\
{$[54]$} & kNN & 100 & $85.7 \%$ accuracy, $\%$ EER \\
{$[55]$} & SVM & 14 & $92.7 \%$ accuracy \\
{$[56]$} & SVM & 51 & $53 \%$ accuracy, $33.3 \%$ EER \\
{$[57]$} & CNN & 4007 & $91 \%$ accuracy, $33.3 \%$ EER \\
{$[59]$} & CC & 15 & $95 \%$ accuracy, $5.5 \%$ EER \\
{$[58]$} & CRM & 48 & $53 \%$ accuracy, $21.7 \%$ EER \\
{$[60]$} & GDI & 744 & $66.3 \%$ accuracy, $5.6 \%$ EER \\
{$[61]$} & GDI & 51 & $37 \%$ accuracy, $7.22 \%$ EER \\
\hline
\end{tabular}

\subsubsection{Touch Dynamics}

Touch dynamics are commonly used authentication methods for smart devices, where touch screens are used as a source for data collection. User authentication is performed by analyzing user behaviors such as gestures, swipes, or tapping on the screen. SaeBae et al. [62] presented a multi-touch gesture-based authentication approach by using five-finger touch gestures and movements that was tested on 34 participants. The authors utilized the Dynamic Time Warping (DTW) algorithm. Their proposed method achieved an accuracy of $90 \%$ with an ERR of 2-5\%. Rauen et al. [63] utilized gesture-related data to verify users. Their method monitors different gesture activities such as how users deal with screen (pressing a button and scrolling styles). They tested their method with a random forest (RF) classification algorithm and achieved an accuracy between 99.68 and $96.26 \%$ with $3.15 \%$ FAR and $9.13 \%$ FRR. Some other studies also used touch dynamics for continuous authentication [64-71]. A performance comparison for these approaches is presented in Table 5.

Table 5. Touch dynamics.

\begin{tabular}{llll}
\hline Studies & Classification Algorithms & \# Users & Performance \\
\hline$[62]$ & DTW & 34 & $90 \%$ accuracy, 2-5\% EER \\
{$[63]$} & RF & - & $99.68-96.26 \%$ accuracy, 3.15\% FAR, 9.13\% FRR \\
{$[64]$} & DT, RF & 41 & $12.5 \%$ FAR, $1.63 \%$ FRR \\
{$[65]$} & L1 distance & 78 & $77 \%$ accuracy, 6.33-15.40\% EER \\
{$[66]$} & (1NN), DTW & 23 & $90 \%$ accuracy \\
{$[67]$} & MHD & 104 & $92.65-93.96 \%$ accuracy, $1.55-0.31 \%$ EER \\
{$[68]$} & SVM & & $95 \%$ accuracy \\
{$[69]$} & SVM & 40 & $88.5 \%$ accuracy, 5.17\% FRR \\
{$[70]$} & PSO-RBFN & 48 & $60 \%$ accuracy, 2.22\% FAR, 2.54\% FRR, 2.4\% EER \\
{$[71]$} & MLP & 20 & $95.96 \%$ accuracy, 6.94\% FAR, $2.55 \%$ FRR \\
& SVM & 20 & $94.4 \%$ accuracy, 3.7\% FAR, 3.5\% FRR \\
& LR & 20 & $84.3 \%$ accuracy, $13.7 \%$ FAR, $14.6 \%$ FRR \\
{$[72]$} & NB & 20 & $86.7 \%$ accuracy, $14.2 \%$ FAR, $11.5 \%$ FRR \\
{$[73]$} & SVM & 41 & $0-4 \%$ EER \\
{$[74]$} & DTW & 48 & $77 \%$ accuracy, 21\% FAR, 19\% FRR \\
\hline
\end{tabular}




\subsubsection{Stylometry Dynamics}

Every user writes text in a unique style. Stylometric-oriented recognition techniques analyze written texts to identify a user's identity. This mode uses sentence structure and semantics to authenticates users. Brocardo et al. [75] presented user authentication approach that verifies users by their stylometry. They divided texts into several blocks, and extracted features vectors from each block. Basic features are extracted by a combination of lexical words and lexical characters, whereas advanced features are extracted by N-gram analysis. They used the support vector machine (SVM) algorithm on two different datasets (Enron, Twitter), and achieved 9.98-21.45\% EER. Kaur et al. [76] conducted experiments to recognize and analyze specific text activity by written text. They analyzed 3057 tweets with different ML algorithms (SVM, k-NN, RF, MLP). Among these 3057 tweets, their approach identified $94.38 \%$ accurately. A performance analysis of a few more studies is discussed in Table 6.

Table 6. Stylometry.

\begin{tabular}{llll}
\hline Studies & Classification Algorithms & \# Users & Performance \\
\hline$[75]$ & SVM & datasets & $9.98-21.45 \%$ EER \\
{$[76]$} & SVM & tweets & $94.38 \%$ accuracy \\
{$[77]$} & SVM & 76 & $12.42 \%$ EER \\
{$[78]$} & KNN & dataset & $91 \%$ accuracy, $3.3 \%$ EER \\
{$[79]$} & SVM & 67 & $0.004 \%$ FAR, $0.01 \%$ FRR \\
\hline
\end{tabular}

\subsubsection{Keystroke Dynamics}

Several researchers have proposed keystroke pattern recognition for user authentication. Such techniques analyze individual typing styles on the keyboard based on the assumption that individuals handle keyboards uniquely. By registering keypress events and time duration, patterns of key latency and key-hold time can be obtained. Assuming that users have unique keystroke patterns, this can be considered as a behavioral biometrics mode and be used for recognizing users for continuous authentication.

Joyce et al. [80] introduced user authentication using keystrokes dynamics in 1990. Their proposed method measured and analyzed typing speed. Their experiments were performed on 33 participants who were asked to type a paragraph as a text. Their experiments achieved $0.25 \%$ FAR and $16.36 \%$ FRR. Gascon et al. [81] proposed a keystroke-based continuous authentication technique, where 300 participants typed short sentences on the smartphone. Typing events were recorded to analyze typing motion of the user's fingers. They utilized SVM for matching, and their method achieved 92\% TPR and 1\% FPR.

The performance of the research works in [81-84] are presented in Table 7.

Table 7. Keystrokes Dynamics.

\begin{tabular}{llll}
\hline Studies & Classification Algorithms & \# Users & Performance \\
\hline$[80]$ & - & 33 & $0.25 \%$ FAR, 16.36\% FRR \\
{$[81]$} & SVM & 300 & $92 \%$ TPR, 1\% FPR \\
{$[82]$} & kNN & 20 & $0.08 \%$ EER \\
{$[83]$} & SVM & 24 & $0.44-3.93 \%$ EER \\
{$[84]$} & kNN & 20 & $97.90 \%$ accuracy, 5.1\% EER \\
{$[85]$} & kNN & 63 & $83.22-92.14 \%$ Accuracy \\
{$[86]$} & Statistical & 100 & $5.73 \%$ FAR, 7.27\% FRR, $6.9 \%$ EER \\
\hline
\end{tabular}

\subsubsection{Eye Movement}

User behavioral features related to eye movements or eye blinks can also be utilized for continuous authentication. In 2004, Kasprowski et al. [87] introduced user authentication based on eye movements. They captured eye fixation on the object (middle and on eight 
edges) with the help of eye-tracking equipment. The experimental results prove that these features are useful for user authentication. Song et al. [41] captured the subjects' focus on the screen and recorded the eye movements. Twenty participants were engaged in experiments; the proposed system achieved $88.73 \%$ accuracy with $10.61 \%$ EER. Further experimental results of a few recent studies [42-44] using eye-movement for continuous authentication are discussed in Table 2. Recently, Saied et al. [45] proposed an eye-blinkbased user authentication system that captures eye-blink patterns and compares them during the authentication phase. They achieved an accuracy of $98.4 \%$, which has been proven on CEW dataset [88]. Experimental results of other studies are presented in Table 8.

Table 8. Eye-movement-based authentication.

\begin{tabular}{lllll}
\hline Studies & Techniques & Classification Algorithms & \# Users & Performance \\
\hline$[41]$ & Eye movement & SVM & 20 & $88.73 \%$ accuracy, 10.61\% EER \\
{$[42]$} & Eye movement & SRC & 30 & $93.1 \%$ accuracy, 6.9\% EER \\
{$[43]$} & Eye movement & SVM & 22 & $3.93 \%$ EER \\
{$[44]$} & Eye movement & SVM & - & $97.95 \%$ accuracy \\
{$[45]$} & Eye blink & CNN & CEW & $98.4 \%$ accuracy \\
\hline$[46]$ & BioAura & SVM, AB & - & $1.9 \%$ EER, 7.6\% FAR, 9.6-8.4\% FRR \\
\hline
\end{tabular}

\subsection{Context-Aware Authentication}

Context-aware modes utilize IP-address, devices, operating systems, and other profiling parameters, such as GPS, battery usage, network usage, web browsing behaviors, and online activities to authenticate a user continuously. Yazji et al. [89] proposed an implicit authentication method by observing user activity patterns to distinguish between normal and abnormal behaviors. Their authentication method monitors user activities, such as the physical location where the files are accessed, which operations are performed on the file, the time when they access the network, and IP addresses of the source and destination. The authors performed experiments on eight users. Their authentication method achieved $90 \%$ accuracy with $13.7 \%$ FAR and $11 \%$ FRR. Gomi et al. [90] proposed browsing-based user recognition for continuous authentication. They collected and analyzed the browsing histories (in conjunction with IP addresses, URLs, and access times) of 1000 users using Linear Regression (LR) to verify the users. The authors achieved $85 \%$ accuracy with $0.03 \%$ EER. Recently, Mahbub et al. [91] utilized user app-usage patterns for continuous authentication. Their method analyzes the time and duration spent on certain applications by a specific user. Based on their analysis, they used hidden Markov models (HMMs) on two datasets (UMDAA-02, Securacy). The performance comparison for these studies is presented in Table 9.

Table 9. Context-aware authentication.

\begin{tabular}{|c|c|c|c|c|}
\hline Studies & Techniques & Classification Algorithms & \# Users & Performance \\
\hline [89] & $\begin{array}{l}\text { File system, network } \\
\text { access, GPS }\end{array}$ & NN, ED & 8 & $\begin{array}{l}90 \% \text { accuracy, }(28.8 \% \text { (file-system), } \\
46.4 \% \text { (networked), } 13.7 \% \text { (combined)) } \\
\text { FAR, }(15.6 \% \text { (file-system) } 7 \% \\
\text { (networked) } 11 \% \text { (combined)) FRR }\end{array}$ \\
\hline [90] & Online activities & LR & Users online history & about $85 \%$ accuracy, $0.03 \%$ EER \\
\hline [91] & App-usage & HMMs & $\begin{array}{l}\text { UMDAA-02, } \\
\text { Securacy }\end{array}$ & $16.16 \%$ EER \\
\hline [92] & Bluetooth, WiFi & K-NN & 200 & $85 \%$ accuracy, $13 \%$ EER \\
\hline [93] & GPS & SVM & MDC Dataset & $82.05 \%$ accuracy \\
\hline [94] & GPS & W2V & CARS, SherLock & $83 \%$ accuracy \\
\hline [95] & GPS & Mot2vec & CDR & not given \\
\hline
\end{tabular}




\section{Security and Privacy Concerns}

This section discusses the criteria for secure and privacy-preserving methods by considering privacy principles [96]. Moreover, this section also discusses security and privacy issues associated with different modes of $\mathrm{CA}$ and possible security vulnerabilities in machine learning (ML) algorithms.

Referring to Question 2, this section discusses how do the different CA modalities score with regard to privacy and security. Privacy issues that are relevant for this paper pertain to the disclosure of person-specific information about behavior, physiological biometric characteristics, and context-aware information, such as location, etc. Security issues relevant for this paper pertain to authentication in the sense of how well the addressed authentication modalities perform. We do not consider software-related security issues, such as software vulnerabilities, nor communication security, etc.

\subsection{Continuous Authentication Cases}

Continuous authentication can be utilized to protect smart devices, such as smartphones, and also cloud-based services. In both scenarios, data can be processed either in the cloud or in the smart device.

Case 1. Authentication processing is performed in the cloud for the purpose of users accessing a cloud-based service. In this case, the device collects data, and continuous data processing and authentication are performed in the cloud. Hence, device processing is reduced, but considerable communication is required, which is consequently powerconsuming. Importantly, this case has privacy issues due to the transmission and revealing of personal data to the cloud.

Case 2a. Authentication processing is performed in the device for the purpose of authenticating the user to the device. So, in this case, the device collects and processes data. The processing requires considerable memory and computational resources, which is power-consuming.

Case $2 b$. Authentication processing is done in the cloud for the purpose of authenticating the user to the mobile device. In this case, the authentication processing is outsourced to a third-party server, which, similarly with Case 1, requires considerable communication. There are, therefore, privacy issues due to the transmission and revealing of personal data to the cloud.

\subsection{Threat Actor Assumptions}

Section 4.1 sketched cases or scenarios for continuous authentication. In this section, we describe relevant threat actor assumptions for these cases.

For Case 1, we assume that there exists a curious (a.k.a. semi-malicious) insider at the server-side who wants to know about the user-specific authentication information, such as location data, IP address, or other online activities, that will be continuously transmitted from the device to the server. We also assume that the curious insider has either partial or full knowledge of training data (i.e., the template) used for physiological or behavioral biometrics, the features computation process, and feature selection criteria. The curious adversary has the capability of template reconstruction. The security issue is that Case 1 relies on the performance of authentication mode. If the adopted authentication mode does not provide good accuracy (i.e., specific mode produces high FAR), then we assume there is a threat of a masquerade attack.

Considering Case 2a in Section 4.1, we assume that the mobile devices securely store user data so that an adversary with access to mobile devices cannot attain the stored templates. The security threat pertains to the performance of the modalities. This is, for instance, of relevance in case a device is stolen, as indicated in Figure $1 b$.

Considering Case $2 \mathrm{~b}$, we assume similar privacy and security threats as mentioned in Case 1, which implies that we have a threat actor that is a malicious or curious insider who has access to authentication data. 


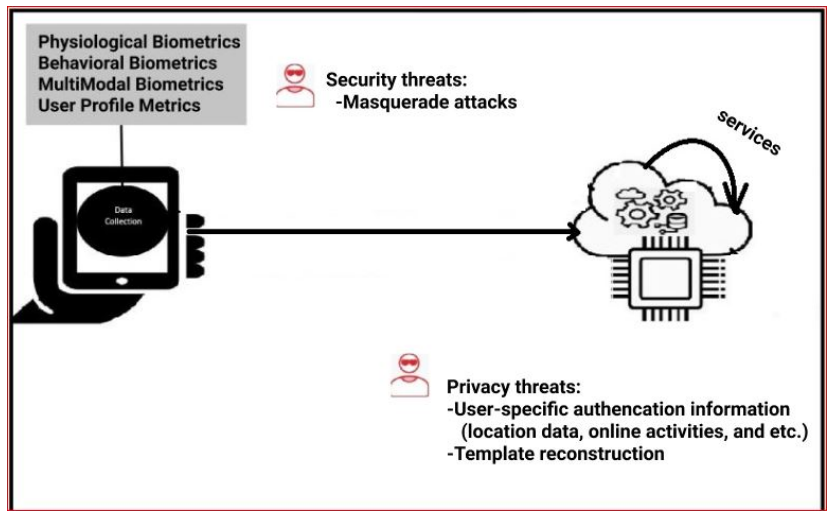

(a) Threats in the cloud

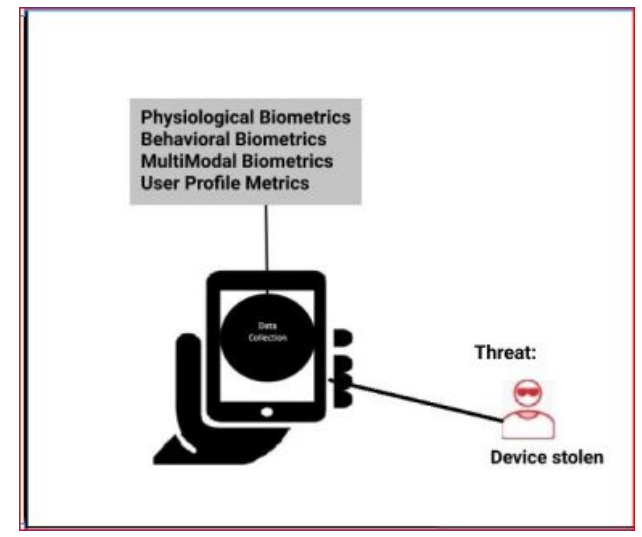

(b) Threats in the device

Figure 1. The threat model.

\subsection{Security Concerns}

The security of continuous authentication modalities is determined by different factors, including the performance such as accuracy, false acceptance rate (FAR), and false rejection rate (FRR) of a specific mode. Secondly, how easy is it to forge a biometric modality, and numbers of possible attacks such as mimicry attacks, template leaking attacks, crosscomparison attacks, etc. Physiological and behavioral biometric authentication mechanisms do not provide 100 percent accuracy, meaning that there are chances of false matches. In general, physiological biometric methods have better accuracy than both behavioral biometrics and context-aware authentication modes. An important point that is often overseen is that these methods are subject to certain kinds of attacks commonly referred to as replay attacks, which, in this context, could be forging fingerprints, etc. [97-102]. Moreover, physiological biometrics need segmentation, which requires more preprocessing. Behavioral biometric-based approaches, such as touch dynamics or keystroke dynamics, can be more efficient because they require less preprocessing compared to physiological biometrics. There are still arguments about whether continuous authentication modes are secure or not. These modes do not provide very good accuracy as these approaches produce a high false acceptance rate (FAR) and false rejection rate (FRR). Due to these reasons, there are possibilities of false acceptance. Moreover, continuous authentication modes with behavioral biometrics are tested on small datasets. These approaches need to be tested on more than one dataset to determine whether these modes produce the same performance, such as same accuracy, same FAR, and FRR on different datasets.

\subsection{Privacy Concerns}

For processing data in the cloud, data are outsourced to the third-part authentication server, which opens security and privacy concerns, i.e., users are not aware of what type of data is collected and stored, how these data will be used in the future, and who has access to their personal data.

Continuous authentication with different modes faces various privacy challenges. Context-aware CA modes monitor user location data obtained by GPS, online user activities, IP address, app-usage, etc. Since these data contain users' personal information, for instance, GPS data reveals the current location of the user. Such techniques cannot protect the privacy of the user's identity and location [103-105].

Continuous authentication by monitoring online activities, such as cookies or online activities, with browsing history data may disclose information about user (such as gender, age, and preferred sites) [106-108]. Researchers [109] performed experiments to identify users by matching anonymous browsing histories with the publicly available dataset (twitter). They achieved more than $70 \%$ accuracy; even browsing history data was in an anonymized form. 
Physiological biometric templates compromise the privacy of user identity information, health information, and other biological information [110]. For instance, CA using face recognition systems to collect and store facial features, which may disclose user emotional states by analyzing facial expressions [111]. Behavioral biometric modalities can also compromise user privacy in similar ways. Behavioral biometric modalities authenticate the user by recognizing their daily life routine data, such as gait recognition, stylometry, touch dynamics, etc., which reveal current user activities. Moreover, keystroke dynamics can be used to identify user age, gender, and the hand used for typing [112]. Compromised profiles based on behavioral biometrics may reveal user identities and behavior that cannot be permanently changed like a password [113].

\subsection{Security and Privacy Challenges in Machine Learning Algorithms}

Machine learning (ML) has several applications in different fields; ML requires continuous collection of high-quality, unprecedented data. These data are uploaded to a centralized location. ML algorithms extract patterns from these data and build models, and models are updated with newly collected data [114]. Physiological and behavioral biometric-based approaches utilize ML algorithms. However, an investigative study [115] provides experimental evidence that $M L$ approaches are vulnerable to sample inference attacks, reconstruction attacks (single and multi-sample), and label distribution estimation attacks (single and multi-sample). A study in [116] performed experiments to prove that ML models are also vulnerable to membership attacks. Moreover, another study [117] also provides common privacy breaches and attacks, such as model inversion, data deanonymization, and model extraction attacks [118].

Machine learning models for classification, such as Support Vector Machine (SVM), k-Nearest Neighbors (kNN), and Hidden Markov Models (HMM), are mostly utilized for various continuous authentication modes. Authors [113] claim that these models (SVM and kNN) store actual user samples in users' authentication profiles. Based on the available data, they utilized positive samples (belong to one user) and presented reconstruction attacks on mobile-based continuous authentication in the cloud, which successfully identifies users from data samples.

\subsection{Attacks on Different Modes of Continuous Authentication}

From a security point of view, continuous authentication with various modes faces different challenges. Countermeasures against various attacks on physiological biometrics have been discussed for decades $[119,120]$, but, still, physiological biometrics are not considered secure authentication modes. Behavioral biometric-based approaches also face distinct security vulnerabilities. Touch dynamics cannot withstand adversarial generative attacks; these attacks manipulate training models to produce erroneous outcomes. Study [121] provides experimental evidence that these attacks on touch dynamics can increase EER ranging from $5 \%$ to $50 \%$. Such attacks on keystroke dynamics can increase EER from $28 \%$ to $84 \%$ [122]. Moreover, Khan et al. [123] demonstrated in experiments on smartphones that keystroke dynamics cannot resist mimicry attacks. Kumar et al. [124] designed imitation attacks on a gait-based authentication system by imitating user gait patterns by using a digital treadmill. Classification results prove that these attacks can increase FAR from 5.8\% to $43.66 \%$. Karimian et al. [125] demonstrated the presentation attack in experiments that if an attacker captures a short template of ECG data by any means (malicious insider), these template data can be used to map attacker ECG data into the victim's ECG data. They collected ECG templates of 52 users from Physikalisch-Technische Bundesanstalt (PTB) database for experiments. Their attacks achieved average success rates of $90 \%$ to $96 \%$.

\section{Usability and Other Issues}

Considering the usability perspective, this section discusses practical challenges associated with the adaptation of continuous authentication (CA). In the context of usability, almost all biometric modalities face distinct challenges [126]. 


\subsection{Modality-Specific Issues}

Regarding Research Question 4, this section discusses the limitations of each modality in different scenarios. In real-life scenarios, the employed authentication modality needs to be determined by the user situation, i.e., what the user is doing (or not doing) at the moment. To the best of our knowledge, none of the single approaches could be suitable for all user situations.

Continuous authentication with physiological biometric-based modalities faces various challenges, such as fingerprint recognition, which requires the user to perform an action (scan fingerprint after some time). Considering the definition for continuous authentication in Section 3, fingerprint recognition conflicts with the concept of continuous authentication because it requires user attention and user action and does not authenticate users passively. Similarly, the voice recognition authentication mode does not fit well with the concept of continuous authentication, as this consequently does not work with quiet users but, in contrast, requires continuous speaking, which is not practical. Moreover, face and iris recognition modes could be utilized for continuous authentication, assuming that the user is holding the device in front of their face. Nevertheless, continuous monitoring with a camera could also affect user acceptance.

Motion-based continuous authentication basically takes the walking style (gait) into account. This implies that in cases of running or jogging, users will not necessarily be recognized or that the recognition accuracy will be lowered.

Some context-aware modes utilize only GPS data to authenticate a user continuously. These approaches are not efficacious when devices are stolen inside a specific area and, in this regard, cannot differentiate whether the user is legitimate or not. Moreover, access is denied to legitimate users when they move out of specified locations. Continuous authentication mode based on online search histories and browsing data does not provide technical details, such as how authentication will work in real scenarios, as continuous authentication requires continuous data. However, it is still unclear how the model will be trained with new data and if users search sites other than their regular routine, how they will be authenticated. Thus, these modes cannot deal with such scenarios, and due to these reasons, these modes are considered weak modes of continuous authentication.

\subsection{Reduced Recognition Accuracy}

User recognition accuracy is important for authentication security in the sense that low accuracy leads to poor authentication security. Likewise, low recognition accuracy in the sense of false rejections affect usability and will be perceived as poor usability and poor user experience [127]. Some behavioral modalities may produce high false acceptance rates (FAR) and false rejection rates (FRR), which consequently will lead to reduced security and usability.

\subsection{Emotional States}

In regard to the previous subsection, a user deals differently with a keyboard or touchscreen during stress compared to their normal mood. Emotional states (such as stress, happiness) will be a factor that also has an impact on recognition accuracy and, therefore, the usability for touch dynamic modalities and behavioral modalities in general.

\subsection{Lack of Standards and Protocols}

A list of standards has been proposed by the international electrotechnical commission (IEC) and the international standard organization (ISO). Usability follows ISO standard 9241-11 [4]; cryptographic authentication protocols follow different ISO standards: entity authentication follows ISO/IEC 9798-3 [128], message authentication using shared key follows ISO 16609:2012 [129], and zero-knowledge proofs and techniques follow ISO/IEC 9798-5 [130], while cybersecurity, information security, and privacy protection follow ISO/IEC JTC 1/SC27 [131]. 
However, we could not find such standards for continuous authentication. It is needed to be standardized, for instance, what estimated time a behavioral biometric-based approach could take to observe user behaviors during the enrollment phase. If the estimated time in the enrollment phase for user behavior observation is too short, then it cannot completely identify a user, which could compromise security. If the estimated enrollment time is too long, then it could affect the usability. The purpose of continuous authentication is to detect imposters immediately after the session begins. The enrollment phase of continuous authentication is different than static authentication; continuous authentication requires more time to observe user behaviors during the enrollment phase [132]. Moreover, the minimum-maximum time to block a device in case of illegitimate access and mechanisms to unblock the device also need to be standardized. Finally, it is also imperative to differentiate that continuous authentication modes, such as behavioral biometrics and context-aware modes, can be used as an identity, or these modes are only utilized for user verification. In general, behavioral biometrics and context-aware modes cannot be used solely as authentication factors; however, these modes could be used as an additional factor with ID/password.

\subsection{Power Consumption Issue}

Continuous authentication actively monitors user actions. Sensors play an essential role, especially continuous authentication with behavioral biometrics. From the data collection phase to authentication and authorization, all processes require sensors and continuous data processing [133]. The deployment and utilization of a certain amount of sensors to improve the recognition accuracy of specific activity, but it requires additional expenses of computation resources and energy consumption. Battery consumption is one of the paramount issues in a smartphone. Smartphones use a number of sensors (e.g., proximity sensors, light sensor, gyroscope, barometer, accelerometer, and a digital compass) [134]. These sensors consume a large amount of battery power. Sensory data are collected at higher power costs [135]. Few studies provide the detailed analysis on smartphone power consumption [136-139]. In general scenarios, power management could be attained by cutting off sensors' power when they are not in use, but continuous authentication requires continuous monitoring and continuous processing, as well as the sensory power that needs to be turned on during the entire active sessions.

\section{Recommendations for Future Research Directions}

This section discusses privacy-preserving approaches and provides recommendations to propose secure and privacy-preserving methods for continuous authentication. Moreover, this section also provides recommendations to improve usability.

\subsection{Privacy-Preserving Approaches}

Continuous authentication with different modes outsources personal data to the server for authentication purposes. Compromised user accounts/profiles can cause identity theft and can also reveal user identity and other related information. These data require secure and privacy-preserving storage and processing. This section discusses privacy-preserving approaches and alludes a few recommendations to achieve secure and privacy-preserving continuous authentication.

\subsubsection{Cancelable Biometrics}

Cancelable biometrics was introduced to solve security and privacy concerns for biometrics. Cancelable biometric approaches provide template non-reversible and biometric salting, which can increase the security and privacy of templates. Images are transformed in a way that makes it difficult to reconstruct the original image from the distorted image. Cancelable Biometrics also provides the capabilities to enroll and revoke new biometric samples, i.e., revoke the previous templates and reissue new templates in case previous templates get compromised. Few studies utilized cancelable biometrics techniques for tem- 
plate protection, such as the authors in [140] utilized random projection approach with the cancelable feature. Authors in the study [141] utilized the fingerprint mixing (mixing two fingerprints) technique. Moreover, a study [142] used a BioHashing interpretation-based cancelable biometric approach to enable privacy.

\subsubsection{Bloom Filters}

A bloom filter [143] is a space-efficient probabilistic data structure of support membership queries. Bloom filters are used to determine whether a given element is a member of a set or not [144]. Bloom filters have intrinsic characteristics that offer advantages, such as the space-efficient, controlled false positive, constant-time query, etc. In recent decades, authors applied bloom filters for biometric templates. The authors in [145] used cancelable biometrics with bloom filters. Moreover, a study [146] used adaptive bloom filters for BTP. Furthermore, the authors in the study [147] also utilized bloom filters to achieve unlinkable and irreversible biometric templates.

\subsubsection{Homomorphic Encryption}

Homomorphic encryption (HE) allows computation on encrypted data so that the data remain confidential during processing. Partially homomorphic encryption (PHE) supports either addition or multiplication at a time. In comparison, fully homomorphic encryption (FHE) supports both operations (addition and multiplication). Thus, by utilizing these homomorphic encryption techniques, the users do not need to trust the server. The users send encrypted data to the server for processing, and the server performs computation without data decryption of the data [148]. During the authentication for services, personal user data are transmitted to an (untrusted) cloud authentication service. Thus, by using homomorphic encryption (HE), we can accomplish data confidentiality. Homomorphic encryption is utilized in a few studies where the biometric data were outsourced to the server. The following studies utilized homomorphic encryption for privacy-preserving biometric authentication: [149-155].

\subsubsection{Secure Two-Party Computation}

In secret sharing schemes, parties share a secret among a group of participants so that no individual can reconstruct the secret from the information available to him. Secret sharing methods enable multiple parties to cooperate with each other and construct/reconstruct the secrets. Secret sharing could be helpful with two-party computation [156] if users do not trust the cloud and do not want to outsource personal data due to privacy concerns.

In the case of the biometric authentication process, users hold their biometric samples, and biometric templates are stored in a database at the server-side. A protocol is executed to determine the similarity or dissimilarity between templates. Secure two-party computation can enable the identification without disclosing biometric data to each other. Thus, utilizing secure two-party computation and dividing the data processing resources between the client and server will be useful to achieve privacy and trust. A list of references that utilized secure two-party computation to achieve privacy: [157-160].

\subsubsection{Zero-Knowledge Proofs}

Zero-Knowledge Proof (ZKP) [161] is considered a privacy-enhancing technique. ZKP enables secure data sharing and ensures that one party can prove itself without disclosing particular or personal information. ZKP does not allow the server to read or write user authentication data, metadata, or cryptographic keys. This technique ensures that user authentication data will remain confidential from malicious or curious insiders and external attackers even if the server gets compromised. The authors in [162] present privacy-preserving authentication with zero-knowledge proofs. 


\subsubsection{Comparison}

Many of the privacy-preserving techniques have been utilized for biometric template protection to solve security and privacy issues. However, these techniques still face distinct challenges. Privacy-preserving methods based on cryptobiometrics, such as fuzzy commitment schemes [163] and fuzzy vault schemes [164], have been utilized for biometric data protection. However, these solutions face issues related to data distinguishability and data reversibility, which cannot provide full privacy [158]. Regarding the performance of cancelable biometric approaches, these approaches can cause two problems: (1) they can obscure the feature of local neighborhoods element, and (2) during the compression phase, alignment cannot be appropriately performed [165].

For privacy-preserving context-aware modes, several statistical privacy techniques, such as $k$-anonymity [166], $l$-Diversity [167], and $t$-closeness [168] can be applied to achieve privacy-preserving continuous authentication. These techniques anonymize user identity attributes, quasi-identifiers, and other sensitive attributes that can reveal the user's identity to achieve privacy. These techniques can also be applied to context-aware data that continuous authentication modes utilize. However, we could not find references related to the application of these approaches in continuous authentication. Experiments can be performed to see what level of privacy and accuracy an authentication system achieves by applying these statistical techniques.

Classical cryptographic approaches demand decryption before comparison, i.e., template comparison cannot be performed in the encrypted domain, implying that templates need to be decrypted during the authentication process. Decryption before authentication can enable an adversary to observe biometric templates and launch an authentication attempt. Homomorphic encryption solves the issue of decryption before authentication [169] because HE allows computation on encrypted data.

Regarding Question 3 in Section 1, and by considering the ISO standard for biometric information protection ISO/IEC 24745 [170], the security and privacy issues for continuous authentication can be mitigated by utilizing cryptographic techniques, such as homomorphic encryption with secure two-party computation and Zero-Knowledge Proofs (ZKP). However, while designing FHE, the degree of polynomials is increased by the addition of noise, which can be result in poor performance. Therefore, FHE requires applying boot-striping for noise removal. Furthermore, bloom filters also seem to be promising techniques to protect biometric information with efficient security and performance. Bloom filters can also be utilized with homomorphic encryption [171]. Continuous authentication modes suffer from significant security and privacy challenges; thus, the utilization of homomorphic encryption combined with bloom filters can solve both security and privacy challenges. Moreover, these techniques can be applied to all modes of continuous authentication, as discussed in Section 3.

\subsection{Recommendations to Improve Usability}

The usability-related issues stated in Section 5 can be improved in different ways. One aspect is to improve the usability with the help of psychology. This includes studying cognitive and social factors, such as user emotions, user behavior, and user habits, to determine the differences in users' emotional states, such as users' behaviors during happiness or anger. This knowledge can be utilized to design new solutions based on users' psychological states that could improve user acceptance and usability. The other way is to ask users' opinions by conducting a survey to know user experiences with different modes of continuous authentication.

Regarding the modality-specific issues discussed in Section 5.1 and in order for continuous authentication to be usable, a set of modalities needs to be considered that could automatically choose the authentication mode according to the scenario. Most of the literature, in general, addresses one or two modalities isolated from other modalities, i.e., these approaches are suitable for only one or two specific situations. Recently, the proposed studies $[172,173]$ utilized multiple modalities and evaluated their approaches on different 
modes. Further work in this direction combined with privacy-preserving approaches can solve modality-specific and privacy issues.

\section{Related Surveys}

This section presents a brief discussion on recently published surveys on continuous authentication (CA), as shown in Table 10. In 2015, the authors presented a survey [174] focusing on a short overview of multi-biometric authentication and discussed the applicability and adoption of implicit authentication with multi-biometric authentication traits. In 2016, Patel et al. [126] presented current progress and future challenges of CA on mobile devices. Ayeswary et al. [175] also presented a brief overview of different CA methods, their merits, and demerits. Moreover, the authors explained open problems and emerging necessities of a continuous authentication system as well. Gonzalez-Manzan et al. [24] presented a comprehensive overview of different components of continuous authentication for the Internet of Things (IoT). Furthermore, this survey also focuses on the industrial status, ongoing research project contributions on continuous authentication, an overview of related standards, and different aspects proposal for future research directions for CA also presented in this survey.

In 2020, Abuhamad et al. [176] presented sensor-based behavioral biometrics, a new survey. This survey describes different behavioral biometric-based approaches and their adoption for CA on smartphones. Rasnayak et al. [177] analyzed continuous authentication from the perspective of usability and resource consumption. In addition, they prepared questionnaires in their survey and asked users' opinions. They conducted a survey involving 500 participants. Furthermore, they showed in their conclusion that users want to utilize continuous authentication, but they want less resource-consuming methods. Furthermore, users have privacy concerns regarding their data that have been utilized for continuous authentication. Eglitis et al. [178] investigated how sensory data are collected and utilized in experiments for behavioral biometrics. Moreover, they examined 32 papers and assessed their citations and how training is performed. Recently, in 2021, the authors of [179] discussed privacy issues associated with sensor-based behavioral biometics. Moreover, they discussed a short overview of behavioral biometric-based approaches. Furthermore, they also presented the review of different available datasets, and finally, the authors also suggested recommendations that could be proven as a considerable privacy-preserving treatment for continuous authentication.

Table 10. A list of continuous authentication surveys.

\begin{tabular}{lcc}
\hline Topics & Year & Focus \\
\hline Expanding continuous authentication with mobile devices [174] & 2015 & CA for IoT \\
\hline $\begin{array}{l}\text { Continuous and transparent multimodal authentication: Reviewing the state of } \\
\text { the art [180] }\end{array}$ & 2016 & CA with multimodal biometrics \\
\hline $\begin{array}{l}\text { Continuous User Authentication on Mobile Devices: Recent Progress and } \\
\text { Remaining Challenges [126] }\end{array}$ & 2016 & CA overview \\
\hline $\begin{array}{l}\text { Continuous Authentication and Authorization for the Internet of Things [181] } \\
\text { Who wants Continuous Authentication on Mobile Devices? [182] }\end{array}$ & 2017 & CA for IoT \\
\hline $\begin{array}{l}\text { Leveraging user-related Internet of Things for continuous authentication: A } \\
\text { survey [24] }\end{array}$ & 2018 & User opinion and experience \\
\hline $\begin{array}{l}\text { A survey on different continuous authentication systems [175] } \\
\text { Data Behind Mobile Behavioural Biometrics-a Survey [178] }\end{array}$ & 2019 & CA for IoT \\
\hline Towards Wider Adoption of Continuous Authentication on Mobile Devices [177] & 2020 & Security and power consumption \\
\hline
\end{tabular}


Table 10. Cont.

\begin{tabular}{lcc}
\hline Topics & Year & Focus \\
\hline $\begin{array}{l}\text { Sensor-based Continuous Authentication of Smartphones' Users Using Behavioral } \\
\text { Biometrics: A Contemporary Survey [176] }\end{array}$ & 2020 & CA with behavioral biometrics \\
\hline $\begin{array}{l}\text { Touch-dynamics based Behavioural Biometrics on Mobile Devices-A Review } \\
\text { from a Usability and Performance Perspective [183] }\end{array}$ & 2020 & Usability and Performance \\
\hline $\begin{array}{l}\text { Privacy-Preserving Sensor-Based Continuous Authentication and User Profiling: } \\
\text { A Review [179] }\end{array}$ & 2021 & Privacy overview \\
\hline
\end{tabular}

\section{Conclusions}

Continuous authentication is slightly different from static authentication. It requires efficient performance in terms of accuracy and high computation. Behavioral biometrics could be the best mode of continuous authentication due to its seamless nature. However, unfortunately, this mode does not achieve very high accuracy yet. Furthermore, other modes of continuous authentication cannot be considered strong modes due to their limitations. Moreover, we cannot ignore other issues related to usability and user experiences before applying continuous authentication in a specific domain. The aspects of security, privacy, and usability in continuous authentication require researchers and industrial attention.

In this survey, we have discussed physiological, behavioral biometrics, and contextaware modes relevant to continuous authentication. We have gathered and compared the results of different studies pertaining to continuous authentication in terms of security, privacy, and usability. Most continuous authentication modes achieve usability to some extent, but security and privacy are still questionable, in which we have identified some security and privacy risks of relevant modes. Moreover, issues related to usability, such as power consumption and lack of standards and protocols, are also identified in this survey. Finally, we have discussed privacy-preserving methods and have provided a comparison and future directions to improve security, privacy, and usability. The recommended improvements can make continuous authentication more applicable in different domains of real-world applications.

Author Contributions: A.F.B. and S.E. contributed equally to this work. All authors have read and agreed to the published version of the manuscript.

Funding: This work is part of the Privacy Matters (PriMa) project. The PriMa project has received funding from European Union's Horizon 2020 research and innovation program under the Marie Skłodowska-Curie grant agreement No. 860315.

Institutional Review Board Statement: Not applicable.

Informed Consent Statement: Not applicable.

Data Availability Statement: Not applicable.

Acknowledgments: Authors would like to express great appreciation to Bian Yang for their valuable comments. Authors also would like to thank all the anonymous reviewers.

Conflicts of Interest: The authors declare no conflict of interest. 


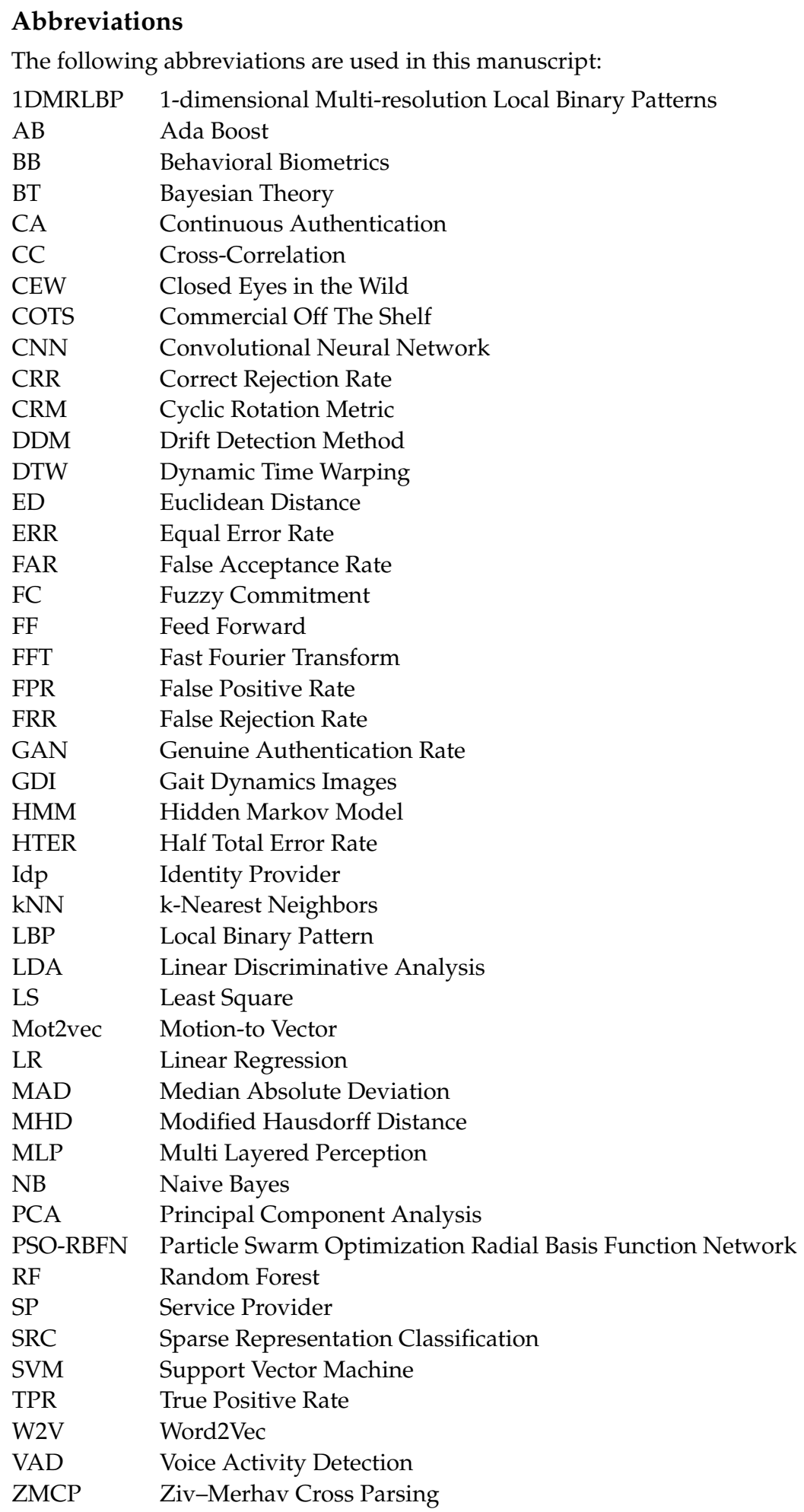

\section{References}

1. Clarke, R. Human identification in information systems. Inf. Technol. People 1994, 7, 6-37. [CrossRef]

2. 2020 Data Breach Investigations Report-Executive Summary. Available online: https://enterprise.verizon.com/resources/ executivebriefs /2020-dbir-executive-brief.pdf (accessed on 15 May 2021).

3. Fenu, G.; Marras, M. Controlling user access to cloud-connected mobile applications by means of biometrics. IEEE Cloud Comput. 2018, 5, 47-57. [CrossRef]

4. ISO/IEC 9241-11:2018. Usability: Definitions and concepts. Available online: https://www.iso.org/obp/ui/iso:std:iso:9241:-11: ed-2:v1:en (accessed on 1 June 2021). 
5. ISO/IEC 24760-1:2019(en). IT Security and Privacy A Framework for Identity Management Part 1: Terminology and Concepts. Available online: https://www.iso.org/obp/ui/iso:std:iso-iec:24760:-1:ed-2:v1:en (accessed on 2 June 2021).

6. Jain, A.K.; Ross, A.; Prabhakar, S. An introduction to biometric recognition. IEEE Trans. Circuits Syst. Video Technol. 2004, 14, 4-20. [CrossRef]

7. Altman, N.S. An introduction to kernel and nearest-neighbor nonparametric regression. Am. Stat. 1992, 46, 175-185.

8. Genesis. Pros and Cons of K-Nearest Neighbors. Available online: https://www.fromthegenesis.com/pros-and-cons-of-knearest-neighbors / (accessed on 22 June 2021).

9. Vapnik, V. Statistical Learning Theory New York; Wiley: New York, NY, USA, 1998.

10. Abeni, P.; Baltatu, M.; D’Alessandro, R. Nis03-4: Implementing biometrics-based authentication for mobile devices. In Proceedings of the IEEE Globecom 2006, San Francisco, CA, USA, 27 November-1 December 2006; pp. 1-5.

11. Crouse, D.; Han, H.; Chandra, D.; Barbello, B.; Jain, A.K. Continuous authentication of mobile user: Fusion of face image and inertial measurement unit data. In Proceedings of the 2015 International Conference on Biometrics (ICB), Phuket, Thailand, 19-22 May 2015; pp. 135-142.

12. Samangouei, P.; Patel, V.M.; Chellappa, R. Facial attributes for active authentication on mobile devices. Image Vis. Comput. 2017, 58, 181-192. [CrossRef]

13. Perera, P.; Patel, V.M. Face-based multiple user active authentication on mobile devices. IEEE Trans. Inf. Forensics Secur. 2018, 14, 1240-1250. [CrossRef]

14. Arora, S. SVM: Difference between Linear and Non-Linear Models. Available online: aitude.com/svm-difference-between-linearand-non-linear-models / (accessed on 21 June 2021).

15. Hansen, L.K.; Salamon, P. Neural network ensembles. IEEE Trans. Pattern Anal. Mach. Intell. 1990, 12, 993-1001. [CrossRef]

16. Bebis, G.; Georgiopoulos, M. Feed-forward neural networks. IEEE Potentials 1994, 13, 27-31. [CrossRef]

17. Rodriguez, P.; Wiles, J.; Elman, J.L. A recurrent neural network that learns to count. Connect. Sci. 1999, 11, 5-40. [CrossRef]

18. Gardner, M.W.; Dorling, S. Artificial neural networks (the multilayer perceptron)-A review of applications in the atmospheric sciences. Atmos. Environ. 1998, 32, 2627-2636. [CrossRef]

19. Hochreiter, S.; Schmidhuber, J. Long short-term memory. Neural Comput. 1997, 9, 1735-1780. [CrossRef]

20. Sakunthala, S.; Kiranmayi, R.; Mandadi, P.N. A review on artificial intelligence techniques in electrical drives: Neural networks, fuzzy logic, and genetic algorithm. In Proceedings of the 2017 International Conference On Smart Technologies For Smart Nation (SmartTechCon), Bengaluru, India, 17-19 August 2017; pp. 11-16.

21. Friedl, M.A.; Brodley, C.E. Decision tree classification of land cover from remotely sensed data. Remote Sens. Environ. 1997, 61, 399-409. [CrossRef]

22. Room, C. Confusion Matrix. Mach. Learn. 2019, 6, 27.

23. Traore, I. Continuous Authentication Using Biometrics: Data, Models, and Metrics: Data, Models, and Metrics; IGI Global: Hershey, PA, USA, 2011.

24. Gonzalez-Manzano, L.; Fuentes, J.M.D.; Ribagorda, A. Leveraging user-related internet of things for continuous authentication: A survey. ACM Comput. Surv. (CSUR) 2019, 52, 1-38. [CrossRef]

25. Feng, H.; Fawaz, K.; Shin, K.G. Continuous authentication for voice assistants. In Proceedings of the 23rd Annual International Conference on Mobile Computing and Networking, Snowbird, UT, USA, 16-20 October 2017; pp. 343-355.

26. Hadid, A.; Heikkila, J.; Silvén, O.; Pietikainen, M. Face and eye detection for person authentication in mobile phones. In Proceedings of the 2007 First ACM/IEEE International Conference on Distributed Smart Cameras, Vienna, Austria, 25-28 September 2007; pp. 101-108.

27. Kudinov, A.A.; Elsakov, S.M. Improved continuous authentication system with counterfeit protection. J. Comput. Eng. Math. 2019, 6, 35-47. [CrossRef]

28. Miguel-Hurtado, O.; Blanco-Gonzalo, R.; Guest, R.; Lunerti, C. Interaction evaluation of a mobile voice authentication system. In Proceedings of the 2016 IEEE International Carnahan Conference on Security Technology (ICCST), Orlando, FL, USA, 24-27 October 2016; pp. 1-8.

29. Zhang, L.; Tan, S.; Yang, J. Hearing your voice is not enough: An articulatory gesture based liveness detection for voice authentication. In Proceedings of the 2017 ACM SIGSAC Conference on Computer and Communications Security, Dallas, TX, USA, 30 October-3 November 2017; pp. 57-71. [CrossRef]

30. Yan, Z.; Zhao, S. A usable authentication system based on personal voice challenge. In Proceedings of the IEEE 2016 International Conference on Advanced Cloud and Big Data (CBD), Chengdu, China, 13-16 August 2016; pp. 194-199.

31. Zhang, L.; Tan, S.; Yang, J.; Chen, Y. Voicelive: A phoneme localization based liveness detection for voice authentication on smartphones. In Proceedings of the 2016 ACM SIGSAC Conference on Computer and Communications Security, Vienna, Austria, 24-28 October 2016; pp. 1080-1091. [CrossRef]

32. Gui, Q.; Ruiz-Blondet, M.V.; Laszlo, S.; Jin, Z. A survey on brain biometrics. ACM Comput. Surv. (CSUR) 2019, 51, 1-38. [CrossRef]

33. Nakanishi, I.; Baba, S.; Miyamoto, C. EEG based biometric authentication using new spectral features. In Proceedings of the IEEE 2009 International Symposium on Intelligent Signal Processing and Communication Systems (ISPACS), Kanazawa, Japan, 7-9 January 2009; pp. 651-654. 
34. Miyamoto, C.; Baba, S.; Nakanishi, I. Biometric person authentication using new spectral features of electroencephalogram (EEG). In Proceedings of the IEEE 2008 International Symposium on Intelligent Signal Processing and Communications Systems, Bangkok, Thailand, 8-11 February 2009; pp. 1-4.

35. Das, R.; Maiorana, E.; Campisi, P. EEG biometrics using visual stimuli: A longitudinal study. IEEE Signal Process. Lett. 2016, 23, 341-345. [CrossRef]

36. Zhang, X.; Yao, L.; Huang, C.; Gu, T.; Yang, Z.; Liu, Y. DeepKey: An EEG and gait based dual-authentication system. arXiv 2017, arXiv:1706.01606.

37. Barra, S.; Casanova, A.; Fraschini, M.; Nappi, M. Fusion of physiological measures for multimodal biometric systems. Multimed. Tools Appl. 2017, 76, 4835-4847. [CrossRef]

38. Louis, W.; Komeili, M.; Hatzinakos, D. Continuous authentication using one-dimensional multi-resolution local binary patterns (1DMRLBP) in ECG biometrics. IEEE Trans. Inf. Forensics Secur. 2016, 11, 2818-2832. [CrossRef]

39. Coutinho, D.P.; Fred, A.L.; Figueiredo, M.A. ECG-based Continuous Authentication System using Adaptive String Matching. In Proceedings of the International Conference on Bio-inspired Systems and Signal Processing (BIOSIGNALS-2011), Rome, Italy, 26-29 January 2011; pp. 354-359. Available online: https:/ / www.scitepress.org/Papers/2011/32920/32920.pdf (accessed on 20 August 2021).

40. Camara, C.; Peris-Lopez, P.; Gonzalez-Manzano, L.; Tapiador, J. Real-time electrocardiogram streams for continuous authentication. Appl. Soft Comput. 2018, 68, 784-794. [CrossRef]

41. Song, C.; Wang, A.; Ren, K.; Xu, W. Eyeveri: A secure and usable approach for smartphone user authentication. In Proceedings of the IEEE INFOCOM 2016-The 35th Annual IEEE International Conference on Computer Communications, San Francisco, CA, USA, 10-14 April 2016; pp. 1-9.

42. Zhang, Y.; Hu, W.; Xu, W.; Chou, C.T.; Hu, J. Continuous authentication using eye movement response of implicit visual stimuli. In Proceedings of the ACM on Interactive Mobile, Wearable and Ubiquitous Technologies; ACM: New York, NY, USA, 2017; Volume 1, pp. 1-22. [CrossRef]

43. Eberz, S.; Lovisotto, G.; Rasmussen, K.B.; Lenders, V.; Martinovic, I. 28 Blinks Later: Tackling Practical Challenges of Eye Movement Biometrics. In Proceedings of the 2019 ACM SIGSAC Conference on Computer and Communications Security, London, UK, 11-15 November 2019; pp. 1187-1199. [CrossRef]

44. Ehatisham-ul Haq, M.; Azam, M.A.; Naeem, U.; Amin, Y.; Loo, J. Continuous authentication of smartphone users based on activity pattern recognition using passive mobile sensing. J. Netw. Comput. Appl. 2018, 109, 24-35. [CrossRef]

45. Saied, M.; Elshenawy, A.; Ezz, M.M. A Novel Approach for Improving Dynamic Biometric Authentication and Verification of Human Using Eye Blinking Movement. Wirel. Pers. Commun. 2020, 115, 859-876. [CrossRef]

46. Mosenia, A.; Sur-Kolay, S.; Raghunathan, A.; Jha, N.K. CABA: Continuous authentication based on BioAura. IEEE Trans. Comput. 2016, 66, 759-772. [CrossRef]

47. Sim, T.; Zhang, S.; Janakiraman, R.; Kumar, S. Continuous verification using multimodal biometrics. IEEE Trans. Pattern Anal. Mach. Intell. 2007, 29, 687-700. [CrossRef]

48. Liwen, F.S.; Cai, X.A.; Ma, J. A dual-biometric-modality identification system based on fingerprint and EEG. In Proceedings of the 2010 Fourth IEEE International Conference on Biometrics: Theory, Applications and Systems (BTAS), Washington, DC, USA, 27-29 September 2010; pp. 1-6.

49. McCool, C.; Marcel, S.; Hadid, A.; Pietikäinen, M.; Matejka, P.; Cernockỳ, J.; Poh, N.; Kittler, J.; Larcher, A.; Levy, C.; et al. Bi-modal person recognition on a mobile phone: Using mobile phone data. In Proceedings of the 2012 IEEE International Conference on Multimedia and Expo Workshops, Melbourne, VIC, Australia, 9-13 July 2012; pp. 635-640.

50. Abo-Zahhad, M.; Ahmed, S.M.; Abbas, S.N. A new multi-level approach to EEG based human authentication using eye blinking. Pattern Recognit. Lett. 2016, 82, 216-225. [CrossRef]

51. Wang, M.; Abbass, H.A.; Hu, J. Continuous authentication using EEG and face images for trusted autonomous systems. In Proceedings of the IEEE 2016 14th Annual Conference on Privacy, Security and Trust (PST), Auckland, New Zealand, 12-14 December 2016; pp. 368-375.

52. Derawi, M.O.; Nickel, C.; Bours, P.; Busch, C. Unobtrusive user-authentication on mobile phones using biometric gait recognition. In Proceedings of the IEEE 2010 Sixth International Conference on Intelligent Information Hiding and Multimedia Signal Processing, Darmstadt, Germany, 15-17 October 2010; pp. 306-311.

53. Mantyjarvi, J.; Lindholm, M.; Vildjiounaite, E.; Makela, S.M.; Ailisto, H. Identifying users of portable devices from gait pattern with accelerometers. In Proceedings of the IEEE International Conference on Acoustics, Speech and Signal Processing (ICASSP'05), Philadelphia, PA, USA, 23 March 2005; Volume 2, pp. p. ii-973.

54. Gafurov, D.; Snekkenes, E. Gait recognition using wearable motion recording sensors. EURASIP J. Adv. Signal Process. 2009, 2009, 1-16. [CrossRef]

55. Hoang, T.; Nguyen, T.D.; Luong, C.; Do, S.; Choi, D. Adaptive Cross-Device Gait Recognition Using a Mobile Accelerometer. J. Inf. Process. Syst. 2013, 9, 333. [CrossRef]

56. Muaaz, M.; Mayrhofer, R. An analysis of different approaches to gait recognition using cell phone based accelerometers. In Proceedings of the International Conference on Advances in Mobile Computing \& Multimedia, Vienna, Austria, 2-4 December 2013; pp. 293-300. [CrossRef] 
57. Wu, Z.; Huang, Y.; Wang, L.; Wang, X.; Tan, T. A comprehensive study on cross-view gait based human identification with deep cnns. IEEE Trans. Pattern Anal. Mach. Intell. 2016, 39, 209-226. [CrossRef]

58. Nickel, C.; Derawi, M.O.; Bours, P.; Busch, C. Scenario test of accelerometer-based biometric gait recognition. In Proceedings of the IEEE 2011 Third International Workshop on Security and Communication Networks (IWSCN), Gjovik, Norway, 18-20 May 2011; pp. 15-21.

59. Sun, Y.; Lo, B. An artificial neural network framework for gait-based biometrics. IEEE J. Biomed. Health Inform. 2018, 23, 987-998. [CrossRef]

60. Zhong, Y.; Deng, Y. Sensor orientation invariant mobile gait biometrics. In Proceedings of the IEEE International Joint Conference on Biometrics, Clearwater, FL, USA, 29 September-2 October 2014; pp. 1-8.

61. Zhong, Y.; Deng, Y.; Meltzner, G. Pace independent mobile gait biometrics. In Proceedings of the 2015 IEEE 7th International Conference on Biometrics Theory, Applications and Systems (BTAS), Arlington, VA, USA, 8-11 September 2015; pp. 1-8.

62. Sae-Bae, N.; Ahmed, K.; Isbister, K.; Memon, N. Biometric-rich gestures: A novel approach to authentication on multi-touch devices. In Proceedings of the SIGCHI Conference on Human Factors in Computing Systems, Austin, TX, USA, 5-10 May 2012; pp. 977-986. [CrossRef]

63. Rauen, Z.I.; Anjomshoa, F.; Kantarci, B. Gesture and sociability-based continuous authentication on smart mobile devices. In Proceedings of the 16th ACM International Symposium on Mobility Management and Wireless Access, Montreal, QC, Canada, 28 October - 2 November 2018; pp. 51-58. [CrossRef]

64. Govindarajan, S.; Gasti, P.; Balagani, K.S. Secure privacy-preserving protocols for outsourcing continuous authentication of smartphone users with touch data. In Proceedings of the 2013 IEEE Sixth International Conference on Biometrics: Theory, Applications and Systems (BTAS), Arlington, VA, USA, 29 September-2 October 2013; pp. 1-8.

65. Zhao, X.; Feng, T.; Shi, W.; Kakadiaris, I.A. Mobile user authentication using statistical touch dynamics images. IEEE Trans. Inf. Forensics Secur. 2014, 9, 1780-1789. [CrossRef]

66. Feng, T.; Yang, J.; Yan, Z.; Tapia, E.M.; Shi, W. Tips: Context-aware implicit user identification using touch screen in uncontrolled environments. In Proceedings of the 15th Workshop on Mobile Computing Systems and Applications, Santa Barbara, CA, USA, 26-27 February 2014; pp. 1-6.

67. Jain, A.; Kanhangad, V. Exploring orientation and accelerometer sensor data for personal authentication in smartphones using touchscreen gestures. Pattern Recognit. Lett. 2015, 68, 351-360. [CrossRef]

68. Holz, C.; Knaust, M. Biometric touch sensing: Seamlessly augmenting each touch with continuous authentication. In Proceedings of the 28th Annual ACM Symposium on User Interface Software \& Technology, Charlotte, NC, USA, 11-15 November 2015; pp. 303-312.

69. Ahmad, J.; Sajjad, M.; Jan, Z.; Mehmood, I.; Rho, S.; Baik, S.W. Analysis of interaction trace maps for active authentication on smart devices. Multimed. Tools Appl. 2017, 76, 4069-4087. [CrossRef]

70. Meng, W.; Wang, Y.; Wong, D.S.; Wen, S.; Xiang, Y. TouchWB: Touch behavioral user authentication based on web browsing on smartphones. J. Netw. Comput. Appl. 2018, 117, 1-9. [CrossRef]

71. Liang, X.; Zou, F.; Li, L.; Yi, P. Mobile terminal identity authentication system based on behavioral characteristics. Int. J. Distrib. Sens. Netw. 2020, 16, 1550147719899371. [CrossRef]

72. Frank, M.; Biedert, R.; Ma, E.; Martinovic, I.; Song, D. Touchalytics: On the applicability of touchscreen input as a behavioral biometric for continuous authentication. IEEE Trans. Inf. Forensics Secur. 2012, 8, 136-148. [CrossRef]

73. De Luca, A.; Hang, A.; Brudy, F.; Lindner, C.; Hussmann, H. Touch me once and i know it's you! implicit authentication based on touch screen patterns. In Proceedings of the SIGCHI Conference on Human Factors in Computing Systems, Austin, TX, USA, 5-10 May 2012; pp. 987-996.

74. Feng, T.; Liu, Z.; Kwon, K.A.; Shi, W.; Carbunar, B.; Jiang, Y.; Nguyen, N. Continuous mobile authentication using touchscreen gestures. In Proceedings of the 2012 IEEE Conference on Technologies for Homeland Security (HST), Waltham, MA, USA, 13-15 November 2012; pp. 451-456.

75. Brocardo, M.L.; Traore, I.; Woungang, I. Authorship verification of e-mail and tweet messages applied for continuous authentication. J. Comput. Syst. Sci. 2015, 81, 1429-1440. [CrossRef]

76. Kaur, R.; Singh, S.; Kumar, H. TB-CoAuth: Text based continuous authentication for detecting compromised accounts in social networks. Appl. Soft Comput. 2020, 97, 106770. [CrossRef]

77. Brocardo, M.L.; Traore, I.; Woungang, I. Toward a framework for continuous authentication using stylometry. In Proceedings of the 2014 IEEE 28th International Conference on Advanced Information Networking and Applications, Victoria, BC, Canada, 13-16 May 2014; pp. 106-115.

78. Saevanee, H.; Clarke, N.; Furnell, S.; Biscione, V. Text-based active authentication for mobile devices. In Proceedings of the IFIP International Information Security Conference, Marrakech, Morocco, 2-4 June 2014; pp. 99-112.

79. Fridman, L.; Stolerman, A.; Acharya, S.; Brennan, P.; Juola, P.; Greenstadt, R.; Kam, M. Multi-modal decision fusion for continuous authentication. Comput. Electr. Eng. 2015, 41, 142-156. [CrossRef]

80. Joyce, R.; Gupta, G. Identity authentication based on keystroke latencies. Commun. ACM 1990, 33, 168-176. [CrossRef]

81. Gascon, H.; Uellenbeck, S.; Wolf, C.; Rieck, K. Continuous authentication on mobile devices by analysis of typing motion behavior. In Proceedings of the Sicherheit 2014-Sicherheit, Schutz und Zuverlässigkeit 2014 Lecture Notes in Informatics (LNI), 
Proceedings-Series of the Gesellschaft fur Informatik (GI), Vienna, Austria, 19-21 March 2014; pp. 1-12. Available online: https:/ / dl.gi.de/handle/20.500.12116/20035?show=full (accessed on 20 August 2021).

82. Giuffrida, C.; Majdanik, K.; Conti, M.; Bos, H. I sensed it was you: Authenticating mobile users with sensor-enhanced keystroke dynamics. In Proceedings of the International Conference on Detection of Intrusions and Malware, and Vulnerability Assessment, Egham, UK, 10-11 July 2014; Springer: Cham, Switzerland, 11 July 2014; pp. 92-111.

83. Cilia, D.; Inguanez, F. Multi-Model authentication using keystroke dynamics for Smartphones. In Proceedings of the 2018 IEEE 8th International Conference on Consumer Electronics-Berlin (ICCE-Berlin), Berlin, Germany, 2-5 September 2018; pp. 1-6.

84. Anusas-Amornkul, T. Strengthening Password Authentication using Keystroke Dynamics and Smartphone Sensors. In Proceedings of the 9th International Conference on Information Communication and Management, Prague, Czech Republic, 23-26 August 2019; pp. 70-74.

85. Monrose, F.; Rubin, A.D. Keystroke dynamics as a biometric for authentication. Future Gener. Comput. Syst. 2000, 16, 351-359. [CrossRef]

86. Chang, T.Y.; Tsai, C.J.; Lin, J.H. A graphical-based password keystroke dynamic authentication system for touch screen handheld mobile devices. J. Syst. Softw. 2012, 85, 1157-1165. [CrossRef]

87. Kasprowski, P.; Ober, J. Eye movements in biometrics. In International Workshop on Biometric Authentication; Springer: Berlin/Heidelberg, Germany, 2004; pp. 248-258.

88. Huang, G.; Ramesh, M.; Berg, T.; Learned-Miller, E. Labeled Faces in the Wild: A Database for Studying Face Recognition in Unconstrained Environments. 2008. Available online: https://hal.inria.fr/inria-00321923/file/Huang_long_eccv2008-lfw.pdf (accessed on 20 August 2021).

89. Yazji, S.; Chen, X.; Dick, R.P.; Scheuermann, P. Implicit user re-authentication for mobile devices. In International Conference on Ubiquitous Intelligence and Computing; Springer: Brisbane, Australia, 9 July 2009; pp. 325-339.

90. Gomi, H.; Yamaguchi, S.; Tsubouchi, K.; Sasaya, N. Continuous authentication system using online activities. In Proceedings of the 2018 17th IEEE International Conference on Trust, Security and Privacy in Computing and Communications/12th IEEE International Conference on Big Data Science and Engineering (TrustCom/BigDataSE), New York, NY, USA, 1-3 August 2018; pp. 522-532.

91. Mahbub, U.; Komulainen, J.; Ferreira, D.; Chellappa, R. Continuous authentication of smartphones based on application usage. IEEE Trans. Biom. Behav. Identity Sci. 2019, 1, 165-180. [CrossRef]

92. Neal, T.J.; Woodard, D.L.; Striegel, A.D. Mobile device application, bluetooth, and wi-fi usage data as behavioral biometric traits. In Proceedings of the 2015 IEEE 7th International Conference on Biometrics Theory, Applications and Systems (BTAS), Arlington, VA, USA, 8-11 September 2015; pp. 1-6.

93. Ying, J.J.C.; Chang, Y.J.; Huang, C.M.; Tseng, V.S. Demographic prediction based on users mobile behaviors. Mob. Data Chall. 2012, 2012, 1-4.

94. Solomon, A.; Bar, A.; Yanai, C.; Shapira, B.; Rokach, L. Predict demographic information using word2vec on spatial trajectories. In Proceedings of the 26th Conference on User Modeling, Adaptation and Personalization, Singapore, 8-11 July 2018; pp. 331-339.

95. Crivellari, A.; Beinat, E. From Motion Activity to Geo-Embeddings: Generating and Exploring Vector Representations of Locations, Traces and Visitors through Large-Scale Mobility Data. ISPRS Int. J. Geo-Inf. 2019, 8, 134. [CrossRef]

96. Langheinrich, M. Privacy by design-Principles of privacy-aware ubiquitous systems. In Proceedings of the International Conference on Ubiquitous Computing, Atlanta, GA, USA, 30 September-2 October 2001; Springer: Cham, Switzerland, 2 October 2001; pp. 273-291.

97. Johnson, R.; Murmuria, R.; Stavrou, A.; Sritapan, V. Pairing continuous authentication with proactive platform hardening. In Proceedings of the 2017 IEEE International Conference on Pervasive Computing and Communications Workshops (PerCom Workshops), Kona, HI, USA, 13-17 March 2017; pp. 88-90.

98. Winder, D. Apple's iPhone FaceID Hacked in Less than 120 Seconds. Available online: https://www.forbes.com/sites/ daveywinder/2019/08/10/apples-iphone-faceid-hacked-in-less-than-120-seconds/?sh=46dfe80521bc (accessed on 10 June 2021).

99. Vaughan-Nichols, S.J. Apple iPhone Fingerprint Reader Confirmed as Easy to Hack. Available online: https://www.zdnet.com/ article/apple-iphone-fingerprint-reader-confirmed-as-easy-to-hack/ (accessed on 3 June 2021).

100. Donohue, B. Lock Screen Bypass Flaw Found in Samsung Androids. Available online: https://threatpost.com/lock-screenbypass-flaw-found-samsung-androids-030413/77580/ (accessed on 30 May 2021).

101. Eden, T. Samsung Lock Screen Security Flaw. Available online: https://shkspr.mobi/blog/2013/03/samsung-lock-screensecurity-flaw / (accessed on 30 May 2021).

102. Racoma, J.A. Android Jelly Bean Face Unlock 'Liveness' Check Easily Hacked with Photo Editing. Available online: https: / / www.androidauthority.com/android-jelly-bean-face-unlock-blink-hacking-105556/ (accessed on 30 May 2021).

103. Vacca, J.R. Computer and Information Security Handbook; Newnes: Waltham, MA, USA, 2012.

104. Kaupins, G.; Minch, R. Legal and ethical implications of employee location monitoring. Int. J. Technol. Hum. Interact. (IJTHI) 2006, 2, 16-35. [CrossRef]

105. Kargl, F.; Lawrence, E.; Fischer, M.; Lim, Y.Y. Security, privacy and legal issues in pervasive ehealth monitoring systems. In Proceedings of the IEEE 2008 7th International Conference on Mobile Business, Barcelona, Spain, 7-8 July 2008; pp. $296-304$. 
106. Smith, M.; Disselkoen, C.; Narayan, S.; Brown, F.; Stefan, D. Browser history re: Visited. In Proceedings of the 12th USENIX Workshop on Offensive Technologies (WOOT 18), Baltimore, MD, USA, 13-14 August 2018.

107. Weinberg, Z.; Chen, E.Y.; Jayaraman, P.R.; Jackson, C. I still know what you visited last summer: Leaking browsing history via user interaction and side channel attacks. In Proceedings of the 2011 IEEE Symposium on Security and Privacy, Oakland, CA, USA, 22-25 May 2011; pp. 147-161.

108. Wondracek, G.; Holz, T.; Kirda, E.; Kruegel, C. A practical attack to de-anonymize social network users. In Proceedings of the 2010 IEEE Symposium on Security and Privacy, Oakland, CA, USA, 16-19 May 2010; pp. 223-238.

109. Su, J.; Shukla, A.; Goel, S.; Narayanan, A. De-anonymizing web browsing data with social networks. In Proceedings of the 26th International Conference on World Wide Web, Perth, Australia, 3-7 April 2017; pp. 1261-1269.

110. Kanade, S.G.; Petrovska-Delacrétaz, D.; Dorizzi, B. Enhancing information security and privacy by combining biometrics with cryptography. Synth. Lect. Inf. Secur. Priv. Trust 2012, 3, 1-140. [CrossRef]

111. Identity Management Institute. Privacy Implications of Using Facial Recognition Systems. Available online: https://www. identitymanagementinstitute.org/privacy-implications-of-using-facial-recognition-systems/ (accessed on 3 June 2021).

112. Roy, S.; Sinha, D.; Roy, U. User authentication: Keystroke dynamics with soft biometric features. Internet Things (IoT) Technol. Appl. Challenges Solut. 2017, 99, 99-118.

113. Al-Rubaie, M.; Chang, J.M. Reconstruction attacks against mobile-based continuous authentication systems in the cloud. IEEE Trans. Inf. Forensics Secur. 2016, 11, 2648-2663. [CrossRef]

114. Al-Rubaie, M.; Chang, J.M. Privacy-preserving machine learning: Threats and solutions. IEEE Secur. Priv. 2019, 17, 49-58. [CrossRef]

115. Salem, A.; Bhattacharya, A.; Backes, M.; Fritz, M.; Zhang, Y. Updates-leak: Data set inference and reconstruction attacks in online learning. In Proceedings of the 29th USENIX Security Symposium (USENIX Security 20), Santa Clara, CA, USA, 12-14 August 2020; pp. 1291-1308.

116. Shokri, R.; Stronati, M.; Song, C.; Shmatikov, V. Membership inference attacks against machine learning models. In Proceedings of the 2017 IEEE Symposium on Security and Privacy (SP), San Jose, CA, USA, 22-26 May 2017; pp. 3-18.

117. Fredrikson, M.; Jha, S.; Ristenpart, T. Model inversion attacks that exploit confidence information and basic countermeasures. In Proceedings of the 22nd ACM SIGSAC Conference on Computer and Communications Security, Denver, CO, USA, 12-16 October 2015; pp. 1322-1333.

118. Tramèr, F.; Zhang, F.; Juels, A.; Reiter, M.K.; Ristenpart, T. Stealing machine learning models via prediction apis. In Proceedings of the 25th USENIX Security Symposium (USENIX Security 16), Austin, TX, USA, 10-12 August 2016; pp. 601-618.

119. Lee, H.; Kwon, T. Fingerprint smudge attacks based on fingerprint image reconstruction on smart devices. J. Korea Inst. Inf. Secur. Cryptol. 2017, 27, 233-240. [CrossRef]

120. Pinto, A.; Pedrini, H.; Krumdick, M.; Becker, B.; Czajka, A.; Bowyer, K.W.; Rocha, A. Counteracting presentation attacks in face, fingerprint, and iris recognition. Deep Learn. Biom. 2018, 245.

121. Serwadda, A.; Phoha, V.V. Examining a large keystroke biometrics dataset for statistical-attack openings. ACM Trans. Inf. Syst. Secur. (TISSEC) 2013, 16, 1-30. [CrossRef]

122. Serwadda, A.; Phoha, V.V. When kids' toys breach mobile phone security. In Proceedings of the 2013 ACM SIGSAC conference on Computer \& Communications Security, Berlin, Germany, 4-8 November 2013; pp. 599-610.

123. Khan, H.; Hengartner, U.; Vogel, D. Mimicry attacks on smartphone keystroke authentication. ACM Trans. Priv. Secur. (TOPS) 2020, 23, 1-34. [CrossRef]

124. Kumar, R.; Phoha, V.V.; Jain, A. Treadmill attack on gait-based authentication systems. In Proceedings of the 2015 IEEE 7th International Conference on Biometrics Theory, Applications and Systems (BTAS), Arlington, VA, USA, 8-11 September 2015; pp. 1-7.

125. Karimian, N.; Woodard, D.L.; Forte, D. On the vulnerability of ecg verification to online presentation attacks. In Proceedings of the 2017 IEEE International Joint Conference on Biometrics (IJCB), Denver, CO, USA, 1-4 October 2017; pp. $143-151$.

126. Patel, V.M.; Chellappa, R.; Chandra, D.; Barbello, B. Continuous user authentication on mobile devices: Recent progress and remaining challenges. IEEE Signal Process. Mag. 2016, 33, 49-61. [CrossRef]

127. Khan, H.; Hengartner, U.; Vogel, D. Usability and security perceptions of implicit authentication: Convenient, secure, sometimes annoying. In Proceedings of the Eleventh Symposium On Usable Privacy and Security (SOUPS 2015), Ottawa, ON, Canada, 22-24 July 2015; pp. 225-239.

128. ISO/IEC 9798-3:2019. IT Security Techniques Entity Authentication Part 3: Mechanisms Using Digital Signature Techniques. Available online: https:/ / www.iso.org/standard/67115.html (accessed on 15 May 2021).

129. ISO 16609:2012. Financial Services Requirements for Message Authentication Using Symmetric Techniques. Available online: https:/ / www.iso.org/standard/55225.html (accessed on 3 June 2021).

130. ISO/IEC 9798-5:2009. Information Technology Security Techniques Entity Authentication Part 5: Mechanisms Using ZeroKnowledge Techniques. Available online: https:/ / www.iso.org/standard/50456.html (accessed on 1 June 2021).

131. Mahmoud, M.S. Information Security, Cybersecurity and Privacy Protection. Available online: https://www.iso.org/committee/ 45306.html (accessed on 1 June 2021).

132. Bours, P. Continuous keystroke dynamics: A different perspective towards biometric evaluation. Inf. Secur. Tech. Rep. 2012, 17, 36-43. [CrossRef] 
133. Rault, T.; Bouabdallah, A.; Challal, Y.; Marin, F. A survey of energy-efficient context recognition systems using wearable sensors for healthcare applications. Pervasive Mob. Comput. 2017, 37, 23-44. [CrossRef]

134. Poza, D. Is FaceID Really Secure? Available online: https://auth0.com/blog/is-faceid-really-secure/ (accessed on 16 June 2021 ).

135. Horvath, Z.; Jenak, I.; Brachmann, F. Battery consumption of smartphone sensors. J. Reliab. Intell. Environ. 2017, 3, 131-136. [CrossRef]

136. Naik, B.A.; Chavan, R. Optimization in power usage of smartphones. Int. J. Comput. Appl. 2015, 119. Available online: https://www.ijcaonline.org/archives/volume119/number18/21165-4224 (accessed on 20 August 2021).

137. Pramanik, P.K.D.; Sinhababu, N.; Mukherjee, B.; Padmanaban, S.; Maity, A.; Upadhyaya, B.K.; Holm-Nielsen, J.B.; Choudhury, P. Power Consumption Analysis, Measurement, Management, and Issues: A State-of-the-Art Review of Smartphone Battery and Energy Usage. IEEE Access 2019, 7, 182113-182172. [CrossRef]

138. Lane, N.D.; Miluzzo, E.; Lu, H.; Peebles, D.; Choudhury, T.; Campbell, A.T. A survey of mobile phone sensing. IEEE Commun. Mag. 2010, 48, 140-150. [CrossRef]

139. Carroll, A.; Heiser, G. An analysis of power consumption in a smartphone. In Proceedings of the USENIX Annual Technical Conference, Boston, MA, USA, 23-25 June 2010; Volume 14, p. 21.

140. Paul, P.P.; Gavrilova, M. Multimodal cancelable biometrics. In Proceedings of the 2012 IEEE 11th International Conference on Cognitive Informatics and Cognitive Computing, Kyoto, Japan, 22-24 August 2012; pp. 43-49.

141. Othman, A.; Ross, A. On mixing fingerprints. IEEE Trans. Inf. Forensics Secur. 2012, 8, 260-267. [CrossRef]

142. Canuto, A.M.; Pintro, F.; Xavier-Junior, J.C. Investigating fusion approaches in multi-biometric cancellable recognition. Expert Syst. Appl. 2013, 40, 1971-1980. [CrossRef]

143. Bloom, B.H. Space/time trade-offs in hash coding with allowable errors. Commun. ACM 1970, 13, 422-426. [CrossRef]

144. Luo, L.; Guo, D.; Ma, R.T.; Rottenstreich, O.; Luo, X. Optimizing Bloom filter: Challenges, solutions, and comparisons. IEEE Commun. Surv. Tutor. 2018, 21, 1912-1949. [CrossRef]

145. Rathgeb, C.; Busch, C. Cancelable multi-biometrics: Mixing iris-codes based on adaptive bloom filters. Comput. Secur. 2014, 42, 1-12. [CrossRef]

146. Rathgeb, C.; Breitinger, F.; Busch, C. Alignment-free cancelable iris biometric templates based on adaptive bloom filters. In Proceedings of the IEEE 2013 International Conference on Biometrics (ICB), Madrid, Spain, 4-7 June 2013; pp. 1-8.

147. Gomez-Barrero, M.; Rathgeb, C.; Galbally, J.; Busch, C.; Fierrez, J. Unlinkable and irreversible biometric template protection based on bloom filters. Inf. Sci. 2016, 370, 18-32. [CrossRef]

148. Gentry, C. Fully homomorphic encryption using ideal lattices. In Proceedings of the Forty-First Annual ACM Symposium on Theory of Computing, Bethesda, MD, USA, 31 May-2 June 2009; pp. 169-178.

149. Gomez-Barrero, M.; Fierrez, J.; Galbally, J.; Maiorana, E.; Campisi, P. Implementation of fixed-length template protection based on homomorphic encryption with application to signature biometrics. In Proceedings of the IEEE Conference on Computer Vision and Pattern Recognition Workshops, Boston, MA, USA, 11-12 June 2015; pp. 191-198.

150. Gomez-Barrero, M.; Maiorana, E.; Galbally, J.; Campisi, P.; Fierrez, J. Multi-biometric template protection based on homomorphic encryption. Pattern Recognit. 2017, 67, 149-163. [CrossRef]

151. Nautsch, A.; Isadskiy, S.; Kolberg, J.; Gomez-Barrero, M.; Busch, C. Homomorphic encryption for speaker recognition: Protection of biometric templates and vendor model parameters. arXiv 2018, arXiv:1803.03559.

152. Steinbauer, M.; Indrawan-Santiago, M.; Anderst-Kotsis, G.; Torres, W.A.A.; Bhattacharjee, N.; Srinivasan, B. Privacy-preserving biometrics authentication systems using fully homomorphic encryption. Int. J. Pervasive Comput. Commun. 2015, 11, 151-168.

153. Halunen, K.; Vallivaara, V. Secure, usable and privacy-friendly user authentication from keystroke dynamics. In Nordic Conference on Secure IT Systems; Springer: Berlin/Heidelberg, Germany, 2016; pp. 256-268.

154. Kolberg, J.; Bauspieß, P.; Gomez-Barrero, M.; Rathgeb, C.; Dürmuth, M.; Busch, C. Template protection based on homomorphic encryption: Computationally efficient application to iris-biometric verification and identification. In Proceedings of the 2019 IEEE International Workshop on Information Forensics and Security (WIFS), Delft, The Netherlands, 9-12 December 2019; pp. 1-6.

155. Morampudi, M.K.; Prasad, M.V.; Verma, M.; Raju, U. Secure and verifiable iris authentication system using fully homomorphic encryption. Comput. Electr. Eng. 2021, 89, 106924. [CrossRef]

156. Yao, A.C.C. How to generate and exchange secrets. In Proceedings of the IEEE 27th Annual Symposium on Foundations of Computer Science (SFCS 1986), Toronto, ON, Canada, 27-29 October 1986; pp. 162-167.

157. Bringer, J.; Favre, M.; Chabanne, H.; Patey, A. Faster secure computation for biometric identification using filtering. In Proceedings of the IEEE 2012 5th IAPR International Conference on Biometrics (ICB), New Delhi, India, 29 March-1 April 2012; pp. 257-264.

158. Bringer, J.; Chabanne, H.; Patey, A. Privacy-preserving biometric identification using secure multiparty computation: An overview and recent trends. IEEE Signal Process. Mag. 2013, 30, 42-52. [CrossRef]

159. Barni, M.; Droandi, G.; Lazzeretti, R. Privacy protection in biometric-based recognition systems: A marriage between cryptography and signal processing. IEEE Signal Process. Mag. 2015, 32, 66-76. [CrossRef]

160. Lin, W.H.; Wu, B.H.; Huang, Q.H. A face-recognition approach based on secret sharing for user authentication in publictransportation security. In Proceedings of the 2018 IEEE International Conference on Applied System Invention (ICASI), Chiba, Japan, 13-17 April 2018; pp. 1350-1353.

161. Goldreich, O.; Oren, Y. Definitions and properties of zero-knowledge proof systems. J. Cryptol. 1994, 7, 1-32. [CrossRef] 
162. Yang, D.; Xu, B.; Yang, B.; Wang, J. A novel biometric authentication scheme with privacy preserving. In Proceedings of the IEEE 2012 Eighth International Conference on Computational Intelligence and Security, Guangzhou, China, 17-18 November 2012; pp. $452-456$.

163. Juels, A.; Wattenberg, M. A fuzzy commitment scheme. In Proceedings of the 6th ACM conference on Computer and communications Security, Singapore, 1-4 November 1999; pp. 28-36.

164. Juels, A.; Sudan, M. A fuzzy vault scheme. Des. Codes Cryptogr. 2006, 38, 237-257. [CrossRef]

165. Rathgeb, C.; Uhl, A. A survey on biometric cryptosystems and cancelable biometrics. EURASIP J. Inf. Secur. 2011, 2011, 1-25. [CrossRef]

166. Samarati, P.; Sweeney, L. Protecting Privacy When Disclosing Information: K-Anonymity and Its Enforcement through Generalization and Suppression. Data Privacy Lab. 1998. Available online: https://dataprivacylab.org/ (accessed on 20 August 2021).

167. Machanavajjhala, A.; Kifer, D.; Gehrke, J.; Venkitasubramaniam, M. L-diversity: Privacy beyond k-anonymity. ACM Trans. Knowl. Discov. Data (TKDD) 2007, 1, 3-es. [CrossRef]

168. Li, N.; Li, T.; Venkatasubramanian, S. t-closeness: Privacy beyond k-anonymity and l-diversity. In Proceedings of the 2007 IEEE 23rd International Conference on Data Engineering, Istanbul, Turkey, 15-20 April 2007; pp. 106-115.

169. Aguilar-Melchor, C.; Fau, S.; Fontaine, C.; Gogniat, G.; Sirdey, R. Recent advances in homomorphic encryption: A possible future for signal processing in the encrypted domain. IEEE Signal Process. Mag. 2013, 30, 108-117. [CrossRef]

170. ISO/IEC 24745:2011. Information Technology Security Techniques BBometric Information Protection. Available online: https: / / www.iso.org/standard/75302.html (accessed on 10 June 2021).

171. Dong, X.; Randolph, D.A. Toward a More Accurate Accrual to Clinical Trials: Joint Cohort Discovery Using Bloom Filters and Homomorphic Encryption. AMIA Summits Transl. Sci. Proc. 2020, 2020, 162.

172. Deb, D.; Ross, A.; Jain, A.K.; Prakah-Asante, K.; Prasad, K.V. Actions speak louder than (pass) words: Passive authentication of smartphone* users via deep temporal features. In Proceedings of the IEEE 2019 International Conference on Biometrics (ICB), Crete, Greece, 4-7 June 2019; pp. 1-8.

173. Brown, J.; Raval, A.; Anwar, M. Towards Passive Authentication using Inertia Variations: An Experimental Study on Smartphones. In Proceedings of the IEEE 2020 Second International Conference on Transdisciplinary AI (TransAI), Irvine, CA, USA, 21-23 September 2020; pp. 88-91.

174. Schaffer, K.B. Expanding continuous authentication with mobile devices. Computer 2015, 48, 92-95. [CrossRef]

175. Ayeswarya, S.; Norman, J. A survey on different continuous authentication systems. Int. J. Biom. 2019, 11, 67-99.

176. Abuhamad, M.; Abusnaina, A.; Nyang, D.; Mohaisen, D. Sensor-based Continuous Authentication of Smartphones' Users Using Behavioral Biometrics: A Survey. arXiv 2020, arXiv:2001.08578. [CrossRef]

177. Rasnayaka, S.; Sim, T. Towards Wider Adoption of Continuous Authentication on Mobile Devices. In Securing Social Identity in Mobile Platforms; Springer: Berlin/Heidelberg, Germany, 2020; pp. 235-262.

178. Eglitis, T.; Guest, R.; Deravi, F. Data Behind Mobile Behavioural Biometrics-A Survey. IET Biom. 2020, 9, 224-237. [CrossRef]

179. Hernández-Álvarez, L.; de Fuentes, J.M.; González-Manzano, L.; Hernández Encinas, L. Privacy-Preserving Sensor-Based Continuous Authentication and User Profiling: A Review. Sensors 2021, $21,92$.

180. Al Abdulwahid, A.; Clarke, N.; Stengel, I.; Furnell, S.; Reich, C. Continuous and transparent multimodal authentication: Reviewing the state of the art. Clust. Comput. 2016, 19, 455-474. [CrossRef]

181. Shahzad, M.; Singh, M.P. Continuous authentication and authorization for the internet of things. IEEE Internet Comput. 2017, 21, 86-90. [CrossRef]

182. Rasnayaka, S.; Sim, T. Who wants Continuous Authentication on Mobile Devices? In Proceedings of the 2018 IEEE 9 th International Conference on Biometrics Theory, Applications and Systems (BTAS), Redondo Beach, CA, USA, 22-25 October 2018; pp. 1-9.

183. Ellavarason, E.; Guest, R.; Deravi, F.; Sanchez-Riello, R.; Corsetti, B. Touch-dynamics based Behavioural Biometrics on Mobile Devices-A Review from a Usability and Performance Perspective. ACM Comput. Surv. (CSUR) 2020, 53, 1-36. [CrossRef] 